\title{
MULTI-DIMENSIONAL SIMULATIONS OF ROTATING PAIR-INSTABILITY SUPERNOVAE
}

\author{
E. Chatzopoulos ${ }^{1}$, J. Craig Wheeler ${ }^{1}$, and Sean M. Couch $^{2,3}$ \\ ${ }^{1}$ Department of Astronomy, University of Texas at Austin, Austin, TX 78712, USA; manolis@astro.as.utexas.edu \\ ${ }^{2}$ Department of Astronomy and Astrophysics, Flash Center for Computational Science, University of Chicago, Chicago, IL 60637, USA \\ Received 2013 June 27; accepted 2013 August 21; published 2013 October 7
}

\begin{abstract}
We study the effects of rotation on the dynamics, energetics, and ${ }^{56} \mathrm{Ni}$ production of pair instability supernova (PISN) explosions by performing rotating two-dimensional ("2.5D") hydrodynamics simulations. We calculate the evolution of eight low-metallicity $\left(Z=10^{-3}, 10^{-4} Z_{\odot}\right)$ massive $\left(135-245 M_{\odot}\right)$ PISN progenitors with initial surface rotational velocities of $50 \%$ of the critical Keplerian value using the stellar evolution code MESA. We allow for both the inclusion and the omission of the effects of magnetic fields in the angular momentum transport and in chemical mixing, resulting in slowly rotating and rapidly rotating final carbon-oxygen cores, respectively. Increased rotation for carbon-oxygen cores of the same mass and chemical stratification leads to less energetic PISN explosions that produce smaller amounts of ${ }^{56} \mathrm{Ni}$ due to the effect of the angular momentum barrier that develops and slows the dynamical collapse. We find a non-monotonic dependence of ${ }^{56} \mathrm{Ni}$ production on rotational velocity in situations when smoother composition gradients form at the outer edge of the rotating cores. In these cases, the PISN energetics are determined by the competition of two factors: the extent of chemical mixing in the outer layers of the core due to the effects of rotation in the progenitor evolution and the development of angular momentum support against collapse. Our 2.5D PISN simulations with rotation are the first presented in the literature. They reveal hydrodynamic instabilities in several regions of the exploding star and increased explosion asymmetries with higher core rotational velocity.
\end{abstract}

Key words: stars: evolution - stars: massive - stars: rotation - supernovae: general - supernovae: individual

Online-only material: color figures

\section{INTRODUCTION}

Pair instability supernovae (PISNe) are triggered by the development of a dynamical instability in the carbon-oxygen $(\mathrm{CO})$ cores of massive stars that enter a regime of high temperature $\left(\sim 10^{9} \mathrm{~K}\right)$ and relatively low density $\left(10^{3}-10^{6} \mathrm{~g} \mathrm{~cm}^{-3}\right)$ favoring the substantial production of electron-positron $\left(e^{+} e^{-}\right)$pairs. When the density of electron-positron pairs becomes high, the volume-averaged adiabatic index decreases $\left(\Gamma_{1}<4 / 3\right)$, eventually triggering dynamical collapse followed by thermonuclear burning of $\mathrm{C}$ and $\mathrm{O}$. This subsonic burning produces enough energy to either totally disrupt the progenitor star (full-fledged PISN) or remove the outermost, less gravitationally bound layers in the form of a pulsational PISN (PPISN; Woosley et al. 2007) depending primarily on the mass of the final CO core, $M_{\mathrm{CO}}$. In addition, large amounts of radioactive ${ }^{56} \mathrm{Ni}\left(\sim 1-60 M_{\odot}\right.$; Heger \& Woosley 2002) are produced, which power the resulting supernova (SN) light curve (LC).

The hypothesis that PISNe are the ultimate fate of very massive stars (VMS, $M_{\text {ZAMS }}>80 M_{\odot}$; ZAMS: zero age main sequence) was introduced more than a half century ago (Rakavy \& Shaviv 1967; Barkat et al. 1967; Rakavy et al. 1967; Fraley 1968). Stellar evolution models of non-rotating PISN progenitors and spherical hydrodynamics simulations of these explosions have been presented in various contexts ever since (Ober et al. 1983; Fryer et al. 2001; Heger \& Woosley 2002). Numerical LCs and spectra for PISNe have also been presented, based on one-dimensional (1D) simulations (Scannapieco et al. 2005; Kasen et al. 2011; Whalen et al. 2012; Dessart et al. 2013). Recently, a few preliminary two-dimensional (2D) simulations of non-rotating PISNe have been presented that investigate the

\footnotetext{
3 Hubble Fellow.
}

effects of mixing due to Rayleigh-Taylor (RT) instabilities that develop between the $\mathrm{CO}$ core and the surrounding He layer of the star and also in the outer regions due to the reverse shock that develops after the SN blast wave exits the stellar surface (Joggerst \& Whalen 2011; Chen et al. 2011, 2012).

Observational evidence suggests the existence of VMS $\left(M_{\text {ZAMS }}<320 M_{\odot}\right)$ capable of producing PISNe (Crowther et al. 2010). In addition, PISNe are thought to be related to some superluminous supernovae (SLSNe; Gal-Yam 2012, and references therein), especially SN 2007bi (Gal-Yam et al. 2009). The nature of SN 2007bi is a topic still under debate, centered around the radiative properties of PISNe (Chatzopoulos \& Wheeler 2012b; Dessart et al. 2013). PPISNe have also been discussed in the context of SLSNe, where the exceptional luminosity is produced by the interaction between multiply ejected PPISN shells (Woosley et al. 2007; Chatzopoulos \& Wheeler 2012b).

The nucleosynthetic output and energetics of PISNe are of great importance to early universe studies where a significant number of Population III stars are found to have masses in the PISN-producing regime in some models (Abel et al. 1998, 2000; Bromm et al. 2002; Bromm \& Larson 2004). Future missions like the James Webb Space Telescope (JWST), WideField Infrared Survey Telescope (WFIRST), and the Wide-Field Infrared Surveyor for High-Redshift are set to look for these primordial PISN explosions that are responsible for enriching the primordial interstellar medium (Scannapieco et al. 2005; Pan et al. 2012; Hummel et al. 2012; Whalen et al. 2012, 2013; de Souza et al. 2013). The number of Population III stars that produce PISNe may be reduced if fragmentation is important (Stacy et al. 2010; Greif et al. 2011), but rapid pre-PISN rotation may counter this effect, allowing for the production of massive CO cores from lower ZAMS mass stars (Chatzopoulos \& Wheeler 2012a; Yoon et al. 2012). Rapid rotation is found to 
be present in star formation simulations of Population III stars (Greif et al. 2011; Stacy et al. 2013).

Woosley et al. (2007) estimated the minimum ZAMS mass limits for PPISNe to be $95 M_{\odot}<M_{\mathrm{ZAMS}}<130 M_{\odot}$ and for PISNe to be $130 M_{\odot}<M_{\text {ZAMS }}<260 M_{\odot}$ in the case of zero rotation and solar metallicity but with an ad hoc pre-PISN massloss assumption. Heger \& Woosley (2002) present these mass limits in terms of the final $M_{\mathrm{CO}}$ (PPISNe: $40 M_{\odot}<M_{\mathrm{CO}}<$ $60 M_{\odot}$, PISNe: $60 M_{\odot}<M_{\mathrm{CO}}<137 M_{\odot}$ ). Langer et al. (2007) estimate that there are no massive stars with $Z>Z_{\odot} / 3$ capable of producing PISNe because of the effects of the extreme mass loss they experience during their evolution.

The ZAMS mass limits for PISNe are reduced if the effects of rotation are taken into account in the progenitor evolution (Chatzopoulos \& Wheeler 2012a; Yoon et al. 2012; Yusof et al. 2013). Rotational mixing, mainly due to meridional circulation but also to the effects of the magnetic field, if considered (Spruit-Tayler dynamo, hereafter "ST"; Spruit 1999, 2002), can recycle unprocessed material from the progenitor's outer envelope into the core, thus increasing the fuel available for nuclear reactions. Rotation in massive stars is found to lead to a bluer, more luminous evolution in the Hertzsprung-Russell diagram and to chemically homogeneous evolution (CHE) in the context of core-collapse supernovae (CCSNe) and gamma-ray burst progenitors (Heger et al. 2000, 2005; Yoon \& Langer 2005; Woosley \& Heger 2006; Ekström et al. 2008, 2012; Maeder \& Meynet 2011; Brott et al. 2011a, 2011b). The CHE has the effect of allowing the production of massive final $\mathrm{CO}$ cores from less massive ZAMS progenitors than required in the case of no rotation. Chatzopoulos \& Wheeler and Yoon et al. derived the mass limits for PPISNe and PISNe in the case of rotating Population III stars to be $\sim 50 M_{\odot}<M_{\mathrm{ZAMS}}<85 M_{\odot}$ for PPISN and $\sim 85 M_{\odot}<M_{\text {ZAMS }}<190 M_{\odot}$ for PISN progenitors rotating at $50 \%$ of the critical Keplerian rate, $\Omega_{c}$, where $\Omega_{c}=\left(g\left(1-\Gamma_{\mathrm{Ed}}\right) / R\right)^{1 / 2}$ with $g=G M / R^{2}$ the gravitational acceleration at the "surface" of the star, $G$ the universal gravitational constant, $M$ the mass, $R$ the radius of the star, and $\Gamma_{\mathrm{Ed}}=L / L_{\mathrm{Ed}}$ the Eddington factor where $L$ and $L_{\mathrm{Ed}}$ are the total radiated luminosity and the Eddington luminosity, respectively. The mechanical effects of the centrifugal force during dynamical collapse can also effect the PISN mass limits: increased rotation can decelerate collapse and even lead to escaping a fullfledged PISN explosion in the most extreme cases (Glatzel et al. 1985). As a result, this shifts the PISN regime to higher mass limits; however, the magnitude of this effect is not established. Rapid rotation $\left(\Omega / \Omega_{c} \geqslant 50 \%\right)$ is observed in nearby massive stars (Dufton et al. 2011).

The effects of rotation on the dynamics of the explosions themselves (the dynamical collapse and the hydrodynamical instabilities that develop, as well as the energetics and nucleosynthetic signature) have not yet been explored in detail, and only a few efforts have been presented in the past in the case of spherical geometry (Glatzel et al. 1985; Stringfellow \& Woosley 1988). Glatzel et al. explored rotation in PISNe using the method of Maclaurin spheroids and found that rigid-body rotation leads to more oblate explosions and less complete explosive oxygen burning. For high degrees of rotation the collapse does not lead to explosion by means of a full-fledged PISN. In the present work, we have used the stellar evolution code MESA (Paxton et al. 2011, 2013) to evolve massive rotating PISN progenitor stars and the new version of the adaptive mesh refinement (AMR) hydrodynamics code FLASH (Fryxell et al. 2000), which includes a treatment for rotation, to study the explosion proper- ties of rotating PISNe in "2.5D" (a 2D grid plus the rotational velocity vectors in the perpendicular direction).

The paper is organized as follows. A presentation of the $M E S A$ pre-PISN progenitor evolution calculations is given in Section 2, the 2.5D FLASH hydrodynamics PISN simulations and results are presented in Section 3, and finally our discussions and conclusions are summarized in Section 4.

\section{PRE-PISN EVOLUTION WITH MESA}

In order to produce physically consistent rotating PISN progenitors for the $2.5 \mathrm{D}$ hydrodynamics simulations, the first step is to evolve a grid of massive stars that produce a variety of final core rotation velocities, $v_{\text {rot,c }}$. The stellar evolution calculations were done with the modular code MESA version 4631 (Paxton et al. 2011, 2013). In all of our MESA calculations, the standard mass-loss rate prescriptions appropriate for massive stars are used (de Jager et al. 1988; Vink et al. 2001). We use the Timmes \& Swesty (2000) "Helmholtz" equation of state (HELM EOS) that includes the contributions from $e^{+} e^{-}$pairs and the "approx 21" nuclear reaction network (Timmes 1999) that includes the $\alpha$-chain elements, and the intermediate elements linking those through $(\alpha, p)(p, \gamma)$ reactions from neutrons and protons all the way up to ${ }^{56} \mathrm{Ni}$ (mass number $A$ from 1 through 56). Those input assumptions are very similar to the ones used for the non-rotating MESA PISN progenitors presented by Dessart et al. (2013). For the treatment of convection, the Schwarzschild criterion is adopted with the choice of $\alpha_{\mathrm{MLT}}=2$ for the mixing length.

Rotation in MESA is treated using the prescriptions of Heger et al. $(2000,2005)$ that include many relevant hydrodynamical instabilities that affect the mixing of chemical species and angular momentum transport (namely, the meridional circulation, the dynamical and secular shear instabilities, and the Solberg-Hoiland and Goldreich-Schubert-Fricke instabilities). MESA also has the capability of including the effects of magnetic fields on angular momentum transport and mixing of species based on the ST prescriptions (Spruit 1999, 2002). The effects of rotation on mass loss are also treated using the approximation presented in Heger et al. (2000): $\dot{M}=\dot{M}_{\text {no-rot }} /\left(1-\Omega / \Omega_{c}\right)^{0.43}$, where $\dot{M}_{\text {no-rot }}$ is the mass-loss rate in the case of no rotation and $\Omega$ is the surface angular velocity at the stellar equator. For cases approaching $\Omega / \Omega_{c}=1$, the mass loss calculated using the above formula diverges. For this reason, in MESA, following Yoon et al. (2010), the mass-loss timescale is limited to the thermal timescale of the star, $\tau_{K H}: \dot{M}=\min \left[\dot{M}(\Omega), f M / \tau_{K H}\right]$, where $f$ is an efficiency factor taken to be 0.3 .

In order to better probe the effects of rotation and to include a variety of progenitor characteristics, we ran eight models that span two metallicity series $\left(Z=10^{-3} Z_{\odot}\right.$ and $\left.Z=10^{-4} Z_{\odot}\right)$ with four models run for each metallicity. The evolution of all models was initiated in the pre-main-sequence phase without rotation up to the ZAMS. Then the desired degree of rotation (50\% of the critical value in all cases) was introduced at the ZAMS for the remainder of the evolution. The evolution was stopped for all models at the same stage of nuclear burning upon encountering the $e^{+} e^{-}$pair instability and at a point where a significant mass fraction of the stellar cores was within the $\Gamma_{1}<4 / 3$ regime in the density-temperature $(\rho-T)$ plane. At this stage, MESA has the capability of computing subsonic hydrodynamical effects and can follow the dynamical collapse up until central ${ }^{20} \mathrm{Ne}$ exhaustion $\left(X_{\mathrm{Ne}, c}=0.01\right.$, where $X_{\mathrm{Ne}, c}$ is the neon mass fraction at the central zone of the model) and 
before ${ }^{16} \mathrm{O}$ burning is initiated, where we formally interrupt the evolution. This is the same criterion used in the PISN progenitor models presented by Ober et al. (1983) and Dessart et al. (2013). Using this termination criterion, we are provided with a set of PISN progenitor models at very similar nuclear burning and hydrodynamical stages that we subsequently map to the AMR grid of FLASH. For all models a high degree of resolution is chosen, resulting in final PISN progenitor models with 4,000-6,000 grid points (the "mesh_delta_coeff" in MESA was given values of $0.35-0.75)$. This reasonable resolution was necessary in order to properly resolve convection and burning processes during the advanced burning stages months to days before the onset of the dynamical instability.

For each metallicity series we ran four different models: (1) a non-rotating model ("norot"), (2) a model with $50 \%$ critical rotation at the ZAMS and the ST effects for the magnetic field included ("rotST"), (3) a model with $50 \%$ critical rotation at the ZAMS with the ST effects omitted ("rotnoST"), and (4) a model with $50 \%$ critical rotation at the ZAMS, the ST effects included, and a higher adopted mass-loss rate parameter used ("rotST_ml2"). The reason that we chose to run models both for the rotST and the rotnoST cases is because we aim to obtain final $\mathrm{CO}$ cores of the same mass but with different rotational profiles. This allows us to study the effects of different degrees of rotation that result from a self-consistent evolution process. The inclusion of magnetic fields ("rotST" models) imposes magnetic torques and magnetic viscosity that can significantly slow down the rotation of the core. The slow core rotation predicted by the ST treatment seems to be consistent with the observed rotation rates of some low-mass stars, but also isolated white dwarfs, suggesting that the cores of some massive stars undergo an angular momentum loss process prior to the explosion (Kawaler 1988; Heger et al. 2005; Suijs et al. 2008). Magnetic braking (Meynet et al. 2011) has been suggested as an alternative explanation for this observation. No direct evidence suggests that the same holds for the cores of VMSs and progenitors of PISNe that experience a much different fate than CCSNe and never reach the burning stages all the way up to Fe. In addition, the efficiency of the ST dynamo mechanism is still much debated (Zahn et al. 2007). This allows us to consider the case where there is no core spin-down process ("rotnoST") and rapidly rotating $\mathrm{CO}$ cores are formed.

We also run "rotST_ml2" models with a higher adopted massloss rate parameter to investigate the competing effects of mass loss and rotationally induced mixing. Increased mass loss during the pre-PISN evolution reduces the smoothing of composition gradients due to rotationally induced mixing at the interface between the $\mathrm{CO}$ core and the outer He envelope. This leads to differences in the dynamical collapse, as we discuss in detail in Section 3. For the hydrodynamical analysis, we chose to include models that do not include the effects of ST, but for which the rotational velocities were artificially set to zero prior to mapping to FLASH ("rotnoST_v0" models) in order to study the effects of rotation in otherwise structurally identical PISN progenitors.

For each metallicity series, the masses of the four models were chosen carefully after a number of trials, so that the resulting final $\mathrm{CO}$ cores had almost identical $M_{\mathrm{CO}}$. The evolution of all models spanned the range 2.5-3.5 Myr. In the case of $Z=$ $10^{-3} Z_{\odot}$ models $200 \mathrm{sm}$ _norot, $140 \mathrm{sm} \_$rotST, $135 \mathrm{sm} \_$rotnoST, and 150sm_rotST_ml2, with ZAMS masses 200, 140, 135, and $150 M_{\odot}$, respectively, all produced final CO cores with $M_{\mathrm{CO}} \simeq$ $80 M_{\odot}$. For $Z=10^{-4} Z_{\odot}$ models $245 \mathrm{sm}$ _norot, 205sm_rotST, $195 \mathrm{sm} \_$rotnoST, and $217 \mathrm{sm} \_$rotST_ml2, with ZAMS masses
245, 205, 195, and $217 M_{\odot}$, respectively, all produced final $\mathrm{CO}$ cores with $M_{\mathrm{CO}} \simeq 100 M_{\odot}$. Therefore all final CO cores have masses deep within the regime predicted for full-fledged PISN explosions. The basic physical characteristics of all MESA models presented here are given in Table 1 (M $M_{\text {ZAMS }}$; the final preexplosion mass $M_{f} ; \Omega / \Omega_{c, X, Y}$, where $X=s, c$ for "surface" and "core edge," respectively, and $Y=i, f$ for the initial (ZAMS) and final pre-PISN values, $v_{\text {rot,c }}$; the total stellar binding energy of the final model, $E_{b, f}$; and the radius $R_{f}$. In all cases the "edge" of the $\mathrm{CO}$ core is defined as the point where the sum of the ${ }^{12} \mathrm{C}$ and ${ }^{16} \mathrm{O}$ mass fractions drop below $\left.0.5, X_{\mathrm{C}}+X_{\mathrm{O}}<0.5\right)$.

The basic structural characteristics of all PISN progenitor models at the time the MESA calculation was terminated are plotted in Figures 1-6. In all figures black curves are for the "norot," red curves for the "rotST," blue curves for the "rotnoST," and green curves for the "rotST_ml2," models. Note how the CO core $\rho, T$ and composition $\left(X_{i}\right)$ structures are strikingly similar for all models of the same metallicity group (the $\mathrm{CO}$ core radii, $R_{\mathrm{CO}}$, range from 0.2 to $1 \times 10^{11} \mathrm{~cm}$ for all models). Exceptions are the rotational (Figures 1, 2, and 5) and radial (Figures 1 and 2) velocity profiles. The rotational velocity profiles show significantly higher rotational velocities for the cores of the "rotnoST" models $\left(>1000 \mathrm{~km} \mathrm{~s}^{-1}\right)$. In retrospect, moderate to low rotational velocities are obtained for the "rotST" and "rotST_ml2" models ( 60-200 $\left.\mathrm{km} \mathrm{s}^{-1}\right)$ and the core-envelope coupling is broken. The radial velocity profiles indicate that at the time of mapping to FLASH the cores of all models are in dynamical collapse with infall velocities exceeding $500 \mathrm{~km} \mathrm{~s}^{-1}$. Although differences of the order of $\sim 100 \mathrm{~km} \mathrm{~s}^{-1}$ exist in the radial velocity profiles between models of the same $M_{\mathrm{CO}}$, we discuss in Section 3 that these differences do not alter the final PISN energetics and ${ }^{56} \mathrm{Ni}$ yields significantly.

Another important thing to note in Figures 3 and 4 is the compositional gradient differences at the interfaces between the $\mathrm{CO}$ cores and the overlying He layers of all models (more specifically at mass coordinates $75-85 M_{\odot}$ for the $Z=$ $10^{-3} Z_{\odot}$ and $95-105 M_{\odot}$ for the $Z=10^{-4} Z_{\odot}$ series). This argument is better illustrated in Section 3.1 in terms of the structure of the mean molecular weight, $\mu$. Non-rotating models produce progenitors with classic, well-defined onion structures between layers with different compositions and clear distinctions between the $\mathrm{CO}$ core and the $\mathrm{H} / \mathrm{He}$ envelope. On the other hand, the core-to-envelope transition for "rotST" and "rotnoST" models generally has smoother composition gradients (specifically for the $\mathrm{He}, \mathrm{C}, \mathrm{N}, \mathrm{O}$, and Ne species). This behavior is reversed again for the "rotST_ml2" models where the rapid mass-loss rate adopted in the evolution calculation prevents effective rotational mixing. This leads to steeper composition gradients in the core-envelope interfaces for the "rotST_ml2" models. Also, the core-envelope compositional transition for the "rotST" models is smoother in the $10^{-3} Z_{\odot}$ model series than in the $10^{-4} Z_{\odot}$ series, indicating that the uncertain effects of mass loss for different degrees of metallicity can significantly affect the ability of a rotating massive star to undergo efficient chemical mixing.

\section{2.5D FLASH SIMULATIONS}

The MESA PISN progenitor models discussed in Section 2 were then mapped to the 2D AMR grid of the multi-physics hydrodynamics code FLASH (Fryxell et al. 2000; Dubey et al. 2009) in order to follow the dynamical collapse, the nucleosynthetic production, and possible hydrodynamic instabilities 


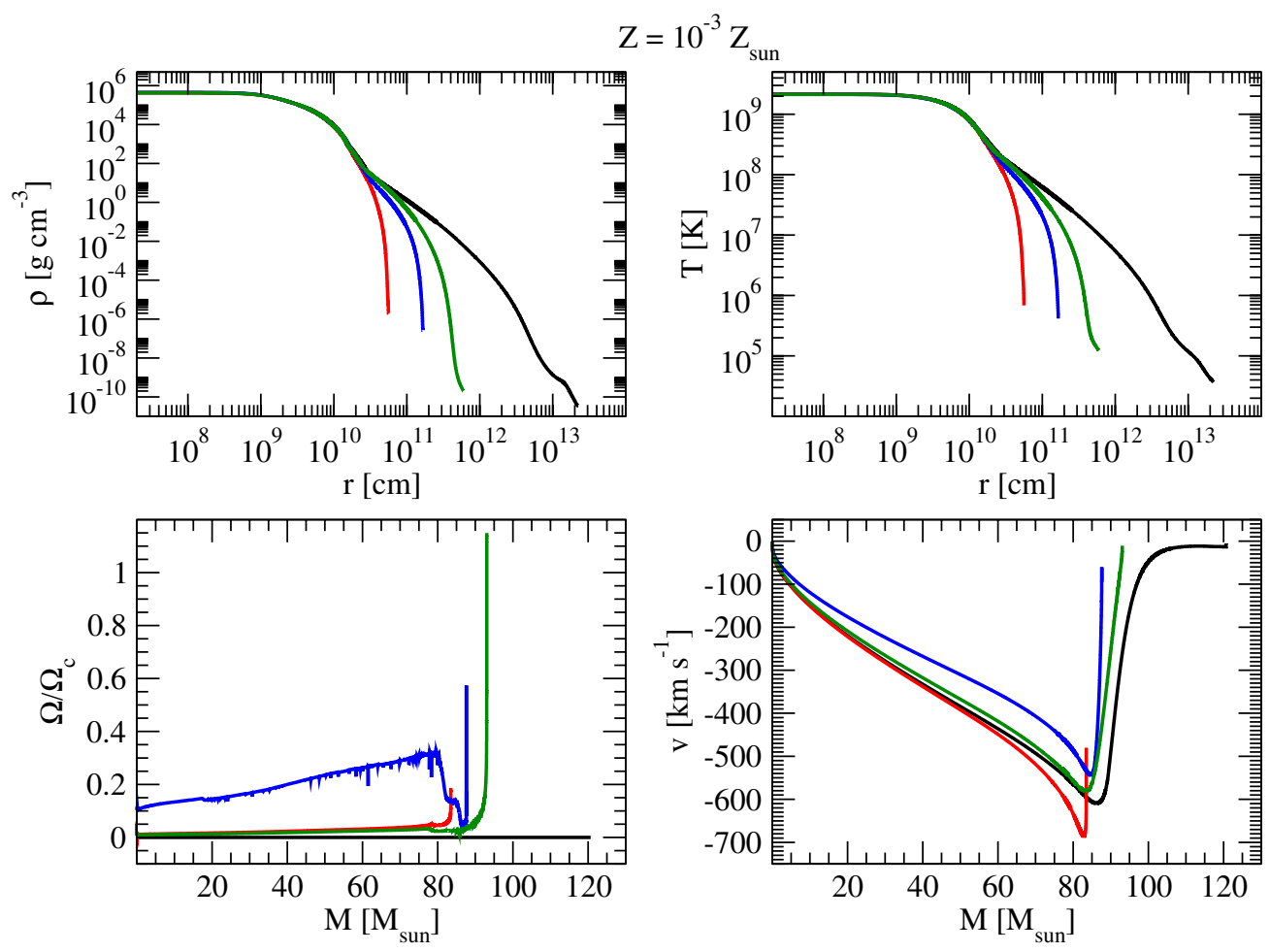

Figure 1. Internal structure plots for the $Z=10^{-3} Z_{\odot} M E S A$ PISN input models at the time of mapping to $F L A S H$. The density and temperature structures are shown in the upper panels, while the internal angular velocity profiles in terms of the critical value are given in the lower left panel and the radial velocity profiles in the lower right panel. Black curves represent the non-rotating ("norot") models, red curves the rotating models with the ST effects included ("rotST"), blue curves the rotating models without the effects of ST ("rotnoST"), and green curves the rotating models with the ST effects included but with a higher mass-loss rate used in the evolution ("rotST_ml2").

(A color version of this figure is available in the online journal.)

Table 1

Characteristics of PISN Progenitor Models

\begin{tabular}{|c|c|c|c|c|c|c|c|c|}
\hline Model & $M_{\text {ZAMS }}$ & $M_{f}$ & $\Omega / \Omega_{c, s, i}$ & $\Omega / \Omega_{c, s, f}$ & $\Omega / \Omega_{c, c, f}{ }^{\mathrm{a}}$ & $v_{\text {rot }, c^{a}}\left(\mathrm{~km} \mathrm{~s}^{-1}\right)$ & $-E_{b, f}\left(10^{53} \mathrm{erg}\right)$ & $R_{f}\left(10^{11} \mathrm{~cm}\right)$ \\
\hline \multicolumn{9}{|c|}{$Z=10^{-3} Z_{\odot}$} \\
\hline 200sm_norot & 200.0 & 120.7 & 0.00 & 0.00 & 0.00 & 0.0 & 1.27 & 249.92 \\
\hline 135sm_rotnoST & 135.0 & 87.6 & 0.50 & 1.00 & 0.30 & 1266.5 & 1.14 & 1.66 \\
\hline $135 \mathrm{sm}$ rotnoST_v0 ${ }^{\mathrm{b}}$ & 135.0 & 87.6 & 0.50 & 0.00 & 0.00 & 0.0 & 1.14 & 1.66 \\
\hline 150sm_rotST_ml2 & 150.0 & 93.1 & 0.50 & 0.99 & 0.03 & 117.4 & 1.18 & 5.99 \\
\hline 205sm_rotST & 205.0 & 123.0 & 0.50 & 0.99 & 0.02 & 90.00 & 1.71 & 8.61 \\
\hline 195sm_rotnoST & 195.0 & 121.5 & 0.50 & 0.13 & 0.25 & 1067.80 & 1.66 & 8.39 \\
\hline 195sm_rotnoST_v0 $0^{\mathrm{b}}$ & 195.0 & 121.5 & 0.50 & 0.00 & 0.00 & 0.00 & 1.66 & 8.39 \\
\hline $217 \mathrm{sm}$ _rotST_ml2 & 217.0 & 122.6 & 0.50 & 0.56 & 0.02 & 65.60 & 1.67 & 9.03 \\
\hline
\end{tabular}

Notes. All masses are expressed in $M_{\odot}$.

a We define as the "edge" of the CO core the radius at which $X_{\mathrm{C}}+X_{\mathrm{O}}<0.5$.

$\mathrm{b}$ The rotational velocities of these pre-PISN models were artificially set to zero for the FLASH hydro simulations in order to investigate the effects of rotation in otherwise identical models.

that develop. The latest release of FLASH (version 4.0) is used with the implementation of the new unsplit piecewise parabolic method hydrodynamic (Lee et al. 2009) solver that allows for the inclusion of angular momentum. The basic physics units implemented in FLASH and used for our simulations are nearly identical to the ones used in MESA (the "Helmholtz" EOS and the "Aprox19" nuclear reaction network). We also use the new and updated Poisson multipole gravity solver.
The mapping from the 1D MESA Lagrangian grid in spherical coordinates to the 2D FLASH Eulerian grid in cylindrical coordinates is done carefully by first converting the cell-outeredge values for radius, $v$ and $v_{\text {rot }}$, in the MESA outputs to cellcenter averaged values. In each case the entire PISN progenitor star is included in the simulation box. A smooth $r^{-2}$ wind with a mass-loss rate $\dot{M}=10^{-5} M_{\odot} \mathrm{yr}^{-1}$ and wind velocity $v_{w}=100 \mathrm{~km} \mathrm{~s}^{-1}$ is joined to the outer edge of all PISN 


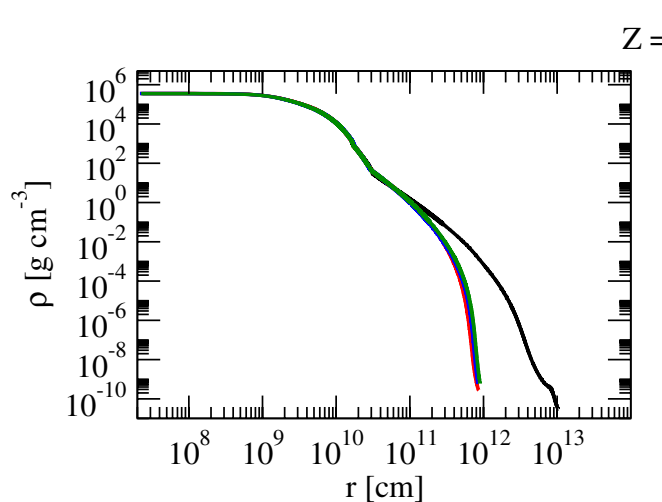

$$
\mathrm{Z}=10^{-4} \mathrm{Z}_{\text {sun }}
$$
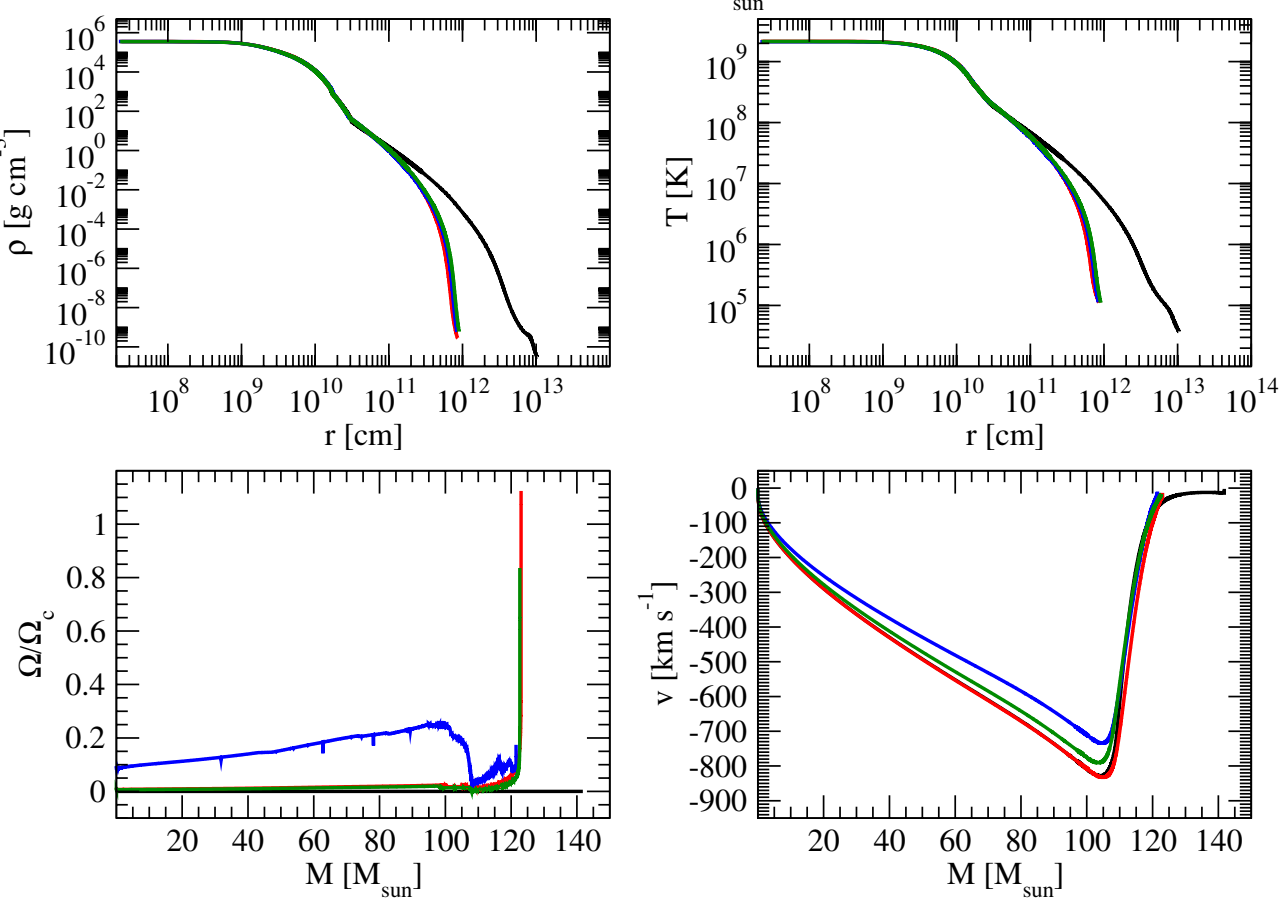

Figure 2. Same as Figure 1 but for the $Z=10^{-4} Z_{\odot} M E S A$ PISN input models.

(A color version of this figure is available in the online journal.)

$$
\mathrm{Z}=10^{-3} \mathrm{Z}_{\text {sun }}
$$

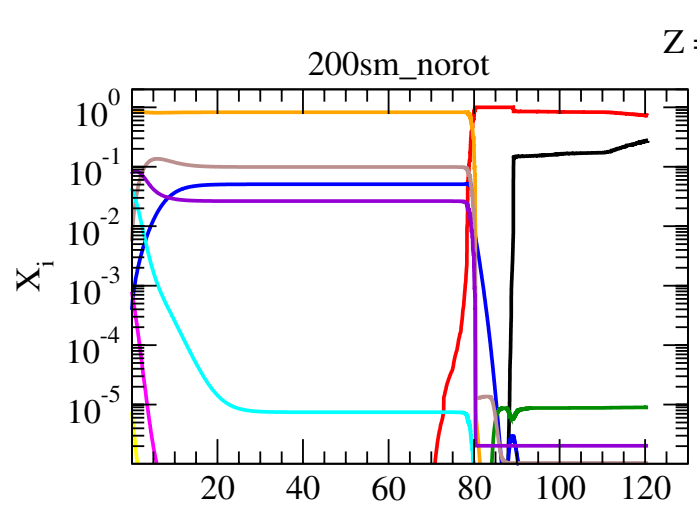

140sm_rotST

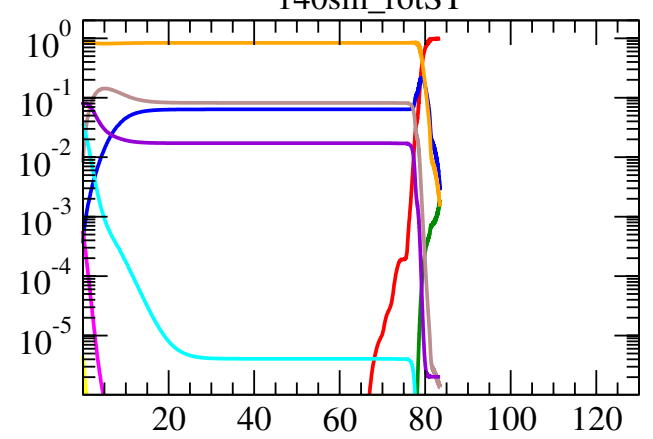

$135 \mathrm{sm}$ rotnoST

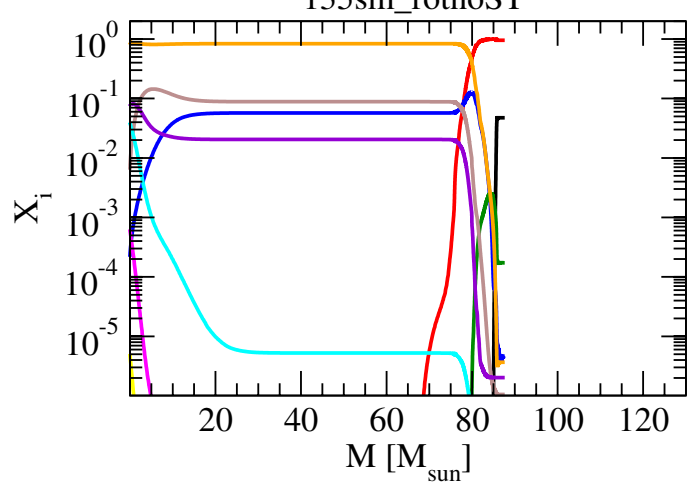

150 sm_rotST_ml2

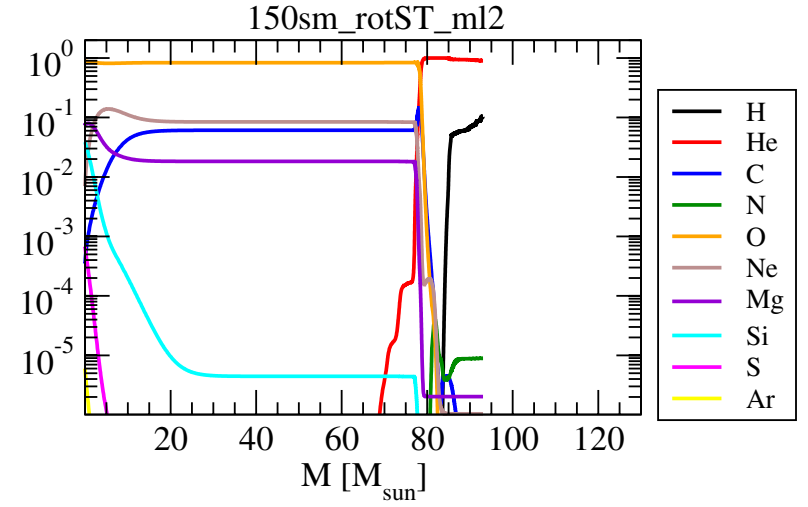

Figure 3. Compositional structures for the $Z=10^{-3} Z_{\odot}$ MESA PISN input models at the time of mapping to FLASH.

(A color version of this figure is available in the online journal.)

progenitors for the FLASH simulations. We then select the proper resolution in FLASH that provides good conservation of total mass, energy, and angular momentum. For the entire duration of the simulations presented here all of these global quantities were found to be conserved at the $\sim 10^{-5}$ level with minor deviations attributable to flows outside of the simulation box and numerical error. Additionally, the grid resolution is chosen carefully in order to properly resolve the ${ }^{12} \mathrm{C}$ and 


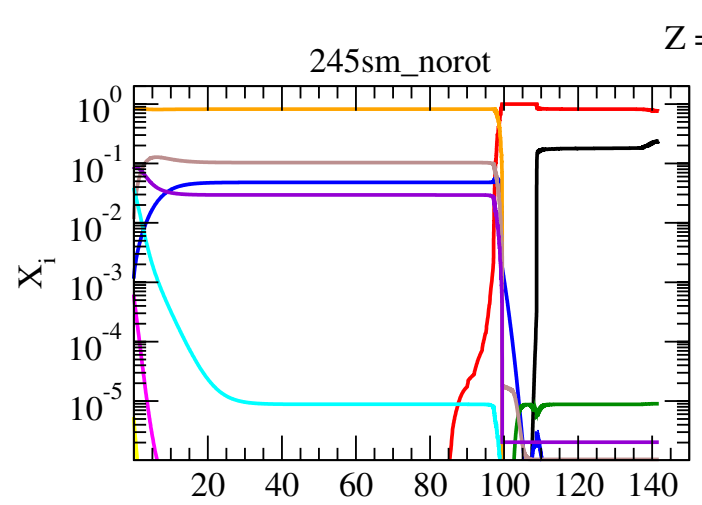

$$
\mathrm{Z}=10^{-4} \mathrm{Z}_{\text {sun }} \quad 205 \mathrm{sm} \text { rotST }
$$
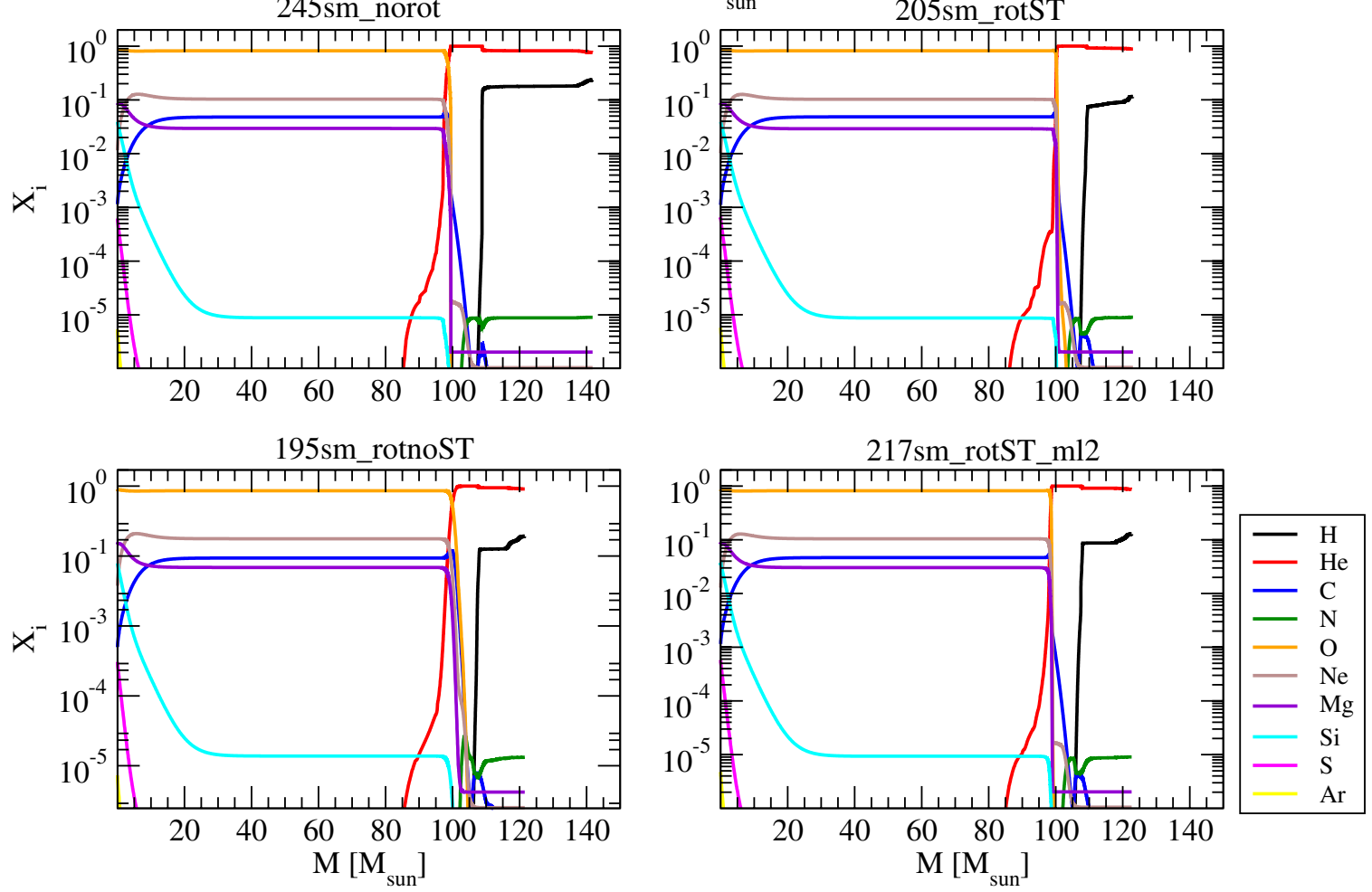

Figure 4. Same as Figure 3 but for the $Z=10^{-4} Z_{\odot}$ MESA PISN input models.

(A color version of this figure is available in the online journal.)

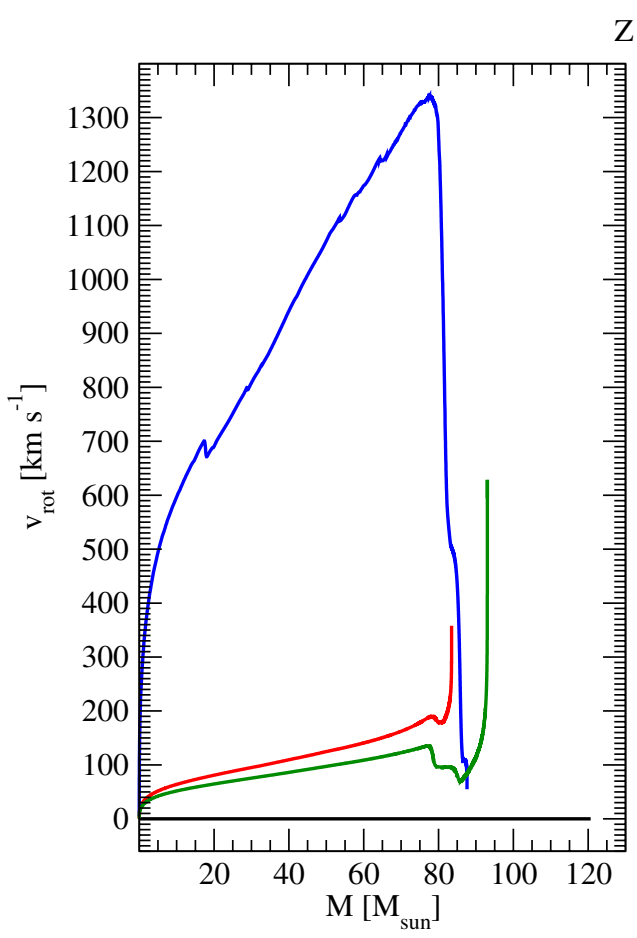

$$
\mathrm{Z}=10^{-3} \mathrm{Z}_{\text {sun }}
$$

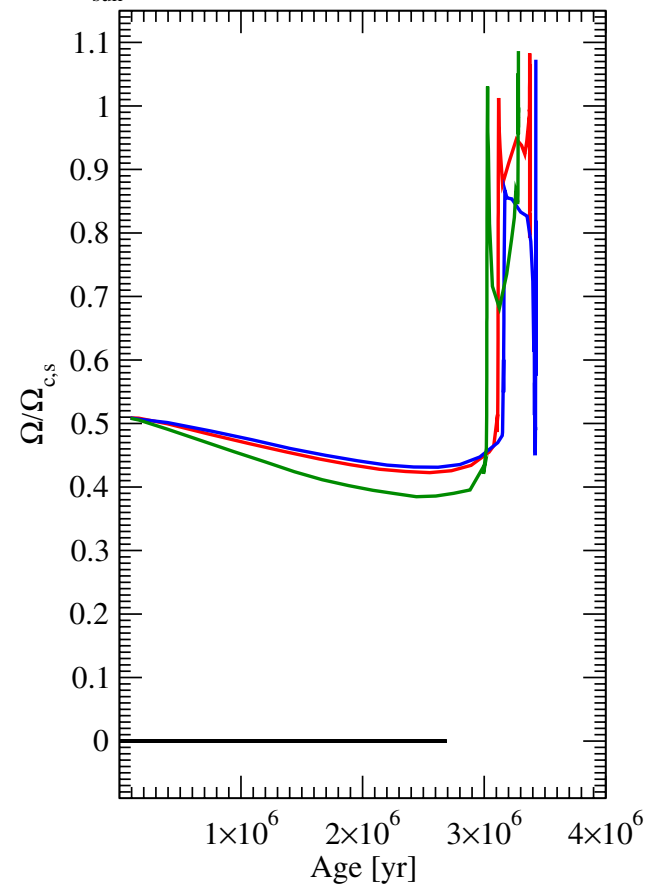

Figure 5. Internal rotational profiles (left) and evolution of $\Omega / \Omega_{c, s}$ (right) for the $Z=10^{-3} Z_{\odot} M E S A$ PISN input models.

(A color version of this figure is available in the online journal.)

${ }^{16} \mathrm{O}$ burning and subsequent flame propagation as well as the hydrodynamical instabilities. For this, a resolution study was performed, yielding scales resolved down to $10^{7}-10^{8} \mathrm{~cm}$ for all models. This spatial resolution is very similar to that suggested by Chen et al. (2011, 2012). In our 2D grids, this resolution corresponds to a total of $10^{6}-10^{7}$ zones, depending on the model simulated. We ran all of our FLASH simulations at the Texas Advanced Computing Center Stampede supercomputer using a 

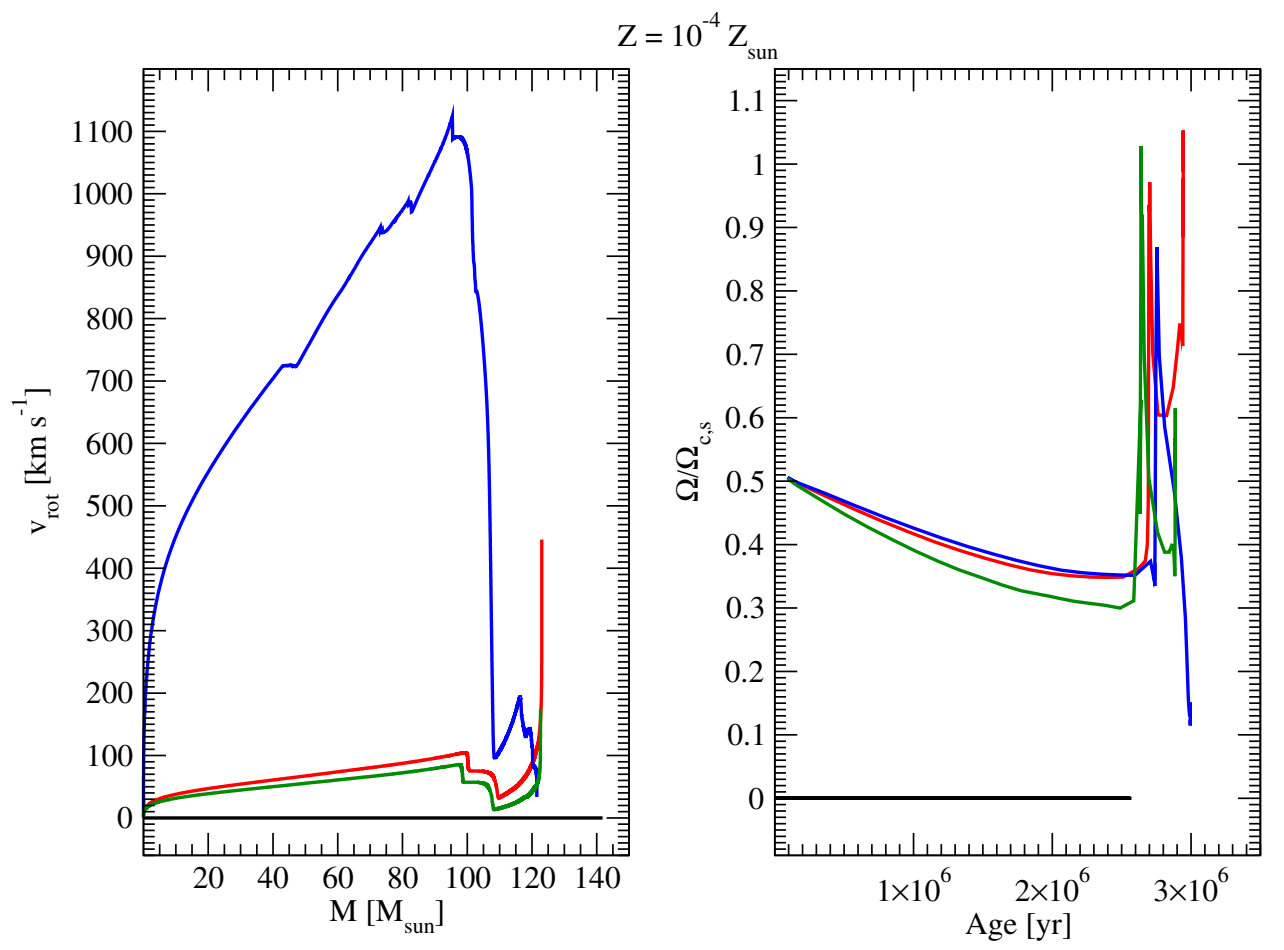

Figure 6. Same as Figure 5 but for the $Z=10^{-4} Z_{\odot} M E S A$ PISN input models.

(A color version of this figure is available in the online journal.)

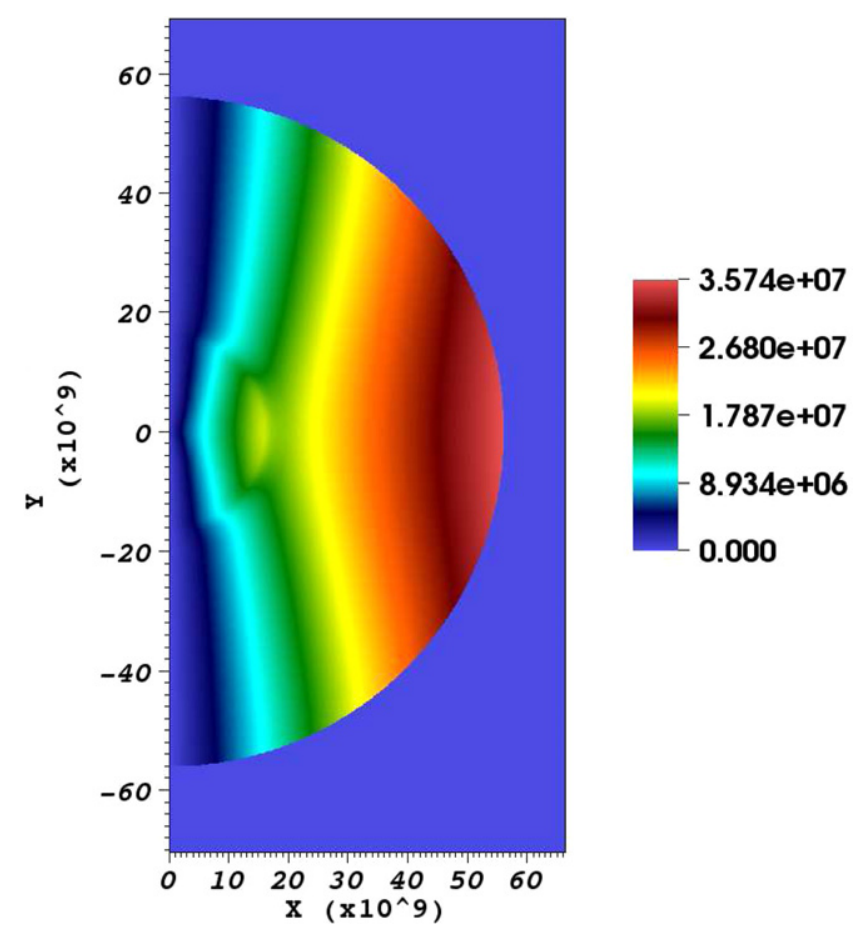

Figure 7. Initial rotation velocity profile of the MESA PISN progenitor model 140sm_rotST mapped in the 2D grid of FLASH assuming shellular rotation. The polar axis (indicated as " $Y$ axis" in the figure) is coincident with the axis of rotation and the rotational velocity vectors point into the plane of the plot. The color-coded legend shows the values for the rotational velocity in $\mathrm{cm} \mathrm{s}^{-1}$.

(A color version of this figure is available in the online journal.)

total of $\sim 10,000$ CPU hours. Visualization of the simulation data was done using the Visit version 2.6 software also run in parallel mode on the Stampede supercomputer.

The rotational velocities computed from $M E S A$ were mapped to the 2D grid of FLASH in cylindrical coordinates as vectors with a direction perpendicular to the $R-z$ plane of the simulation and the rotation axis coincident with the polar axis. To properly account for the treatment of shellular rotation in mapping 1D rotational velocities from $M E S A$ into the 2D grid of $F L A S H$, the following formula was used:

$$
v_{\text {rot }}(R, z)=\Omega(r) R,
$$

where $\Omega(r)=v_{\text {rot }}(r) / r, v_{\text {rot }}(r)$ is the MESA 1D rotational velocity and $r$ is the spherical radial coordinate $\left(r=\left(x^{2}+y^{2}\right)^{0.5}\right)$. Recall that the angular velocity, $\Omega(r)$, is a constant for a particular spherical shell in the shellular approximation. Formally, shellular rotation is defined as constant $\Omega$ on equipotential surfaces (Zahn 1992; Meynet \& Maeder 1997). In the cases we study here, however, the angular velocities in the cores of the model never exceed $40 \%$ of the critical value and the equipotential surfaces are close to spherical. The result of this rotational velocity mapping scheme on the $2 \mathrm{D}$ cylindrical-coordinate grid of FLASH is shown in Figure 7 for the 140sm_rotST model.

The simulations were run until after the SN shock broke out of the stellar surface in each model. For all models, upon mapping to FLASH, the dynamical timescale of collapse was effectively the free-fall timescale $(\sim 50-100 \mathrm{~s})$. The ${ }^{16} \mathrm{O}$ mass fraction and density structures at times of the order of $\sim 100 \mathrm{~s}$ after dynamical collapse are presented in Figures 8-11 for the $10^{-3} Z_{\odot}$ model series and in Figures $12-15$ for the $10^{-4} Z_{\odot}$ model series. The development of RT instability between the oxygen core and the $\mathrm{H} / \mathrm{He}$ envelope is clearly visible for all models with the exception of model 140sm_rotST. The latter is due to the fact that the $\mathrm{H} / \mathrm{He}$ envelope is very thin in this PISN progenitor, and as a result the interaction between the core and envelope material during collapse is weak, suppressing the development of prominent RT fingers.

The appearance of the RT instability throughout the PISN explosions results in mild chemical mixing between the core and envelope material and is in agreement with the findings of 

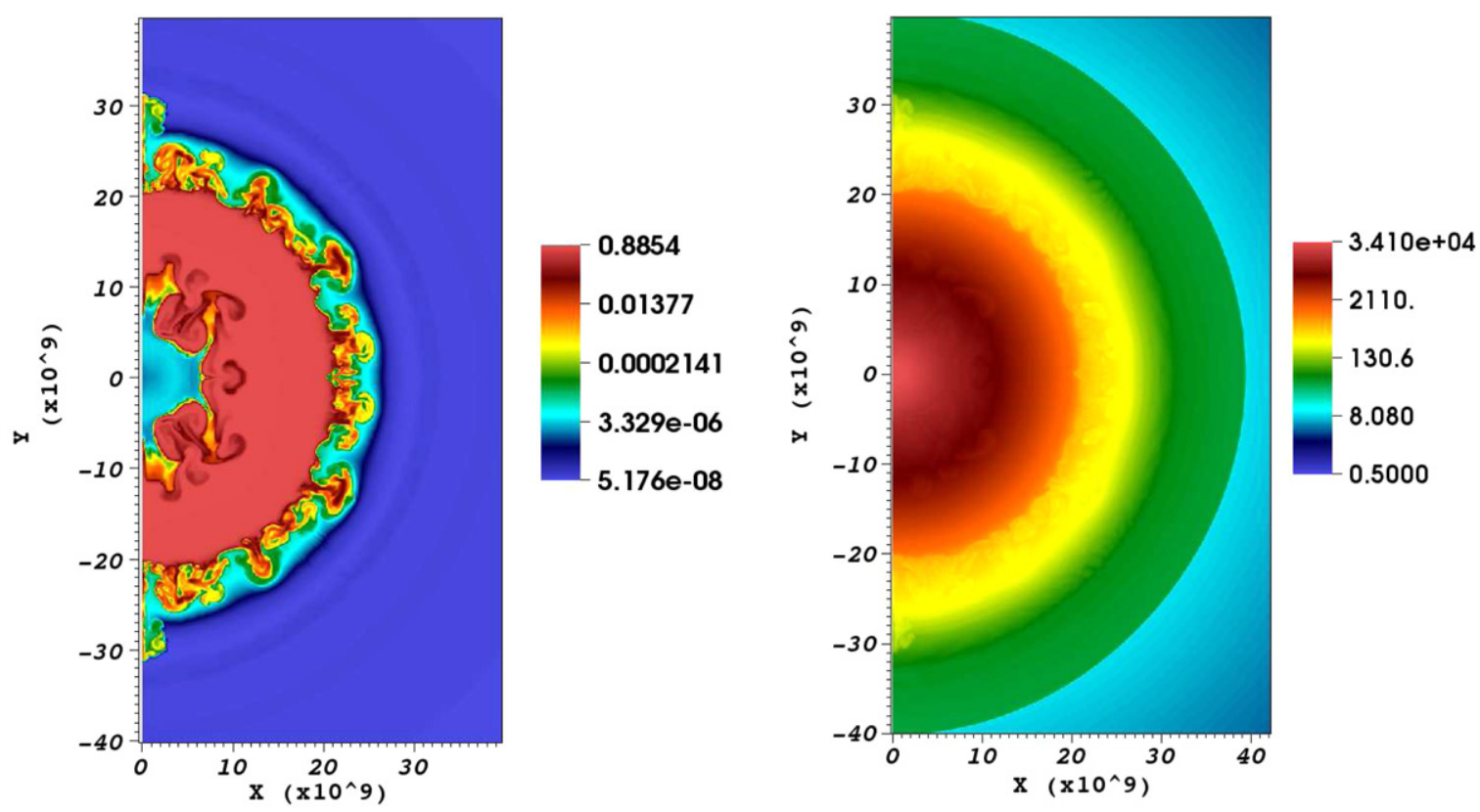

Figure 8. Left: ${ }^{16} \mathrm{O}$ mass fraction of the PISN model 200sm_norot $\left(Z=10^{-3} Z_{\odot}\right)$ at time $t=103 \mathrm{~s}$ after the onset of dynamical collapse. Right: density profile of the same model at the same instant. The color-coded legends show the ${ }^{16} \mathrm{O}$ mass fraction and density in units of $\mathrm{g} \mathrm{cm}^{-3}$.

(A color version of this figure is available in the online journal.)
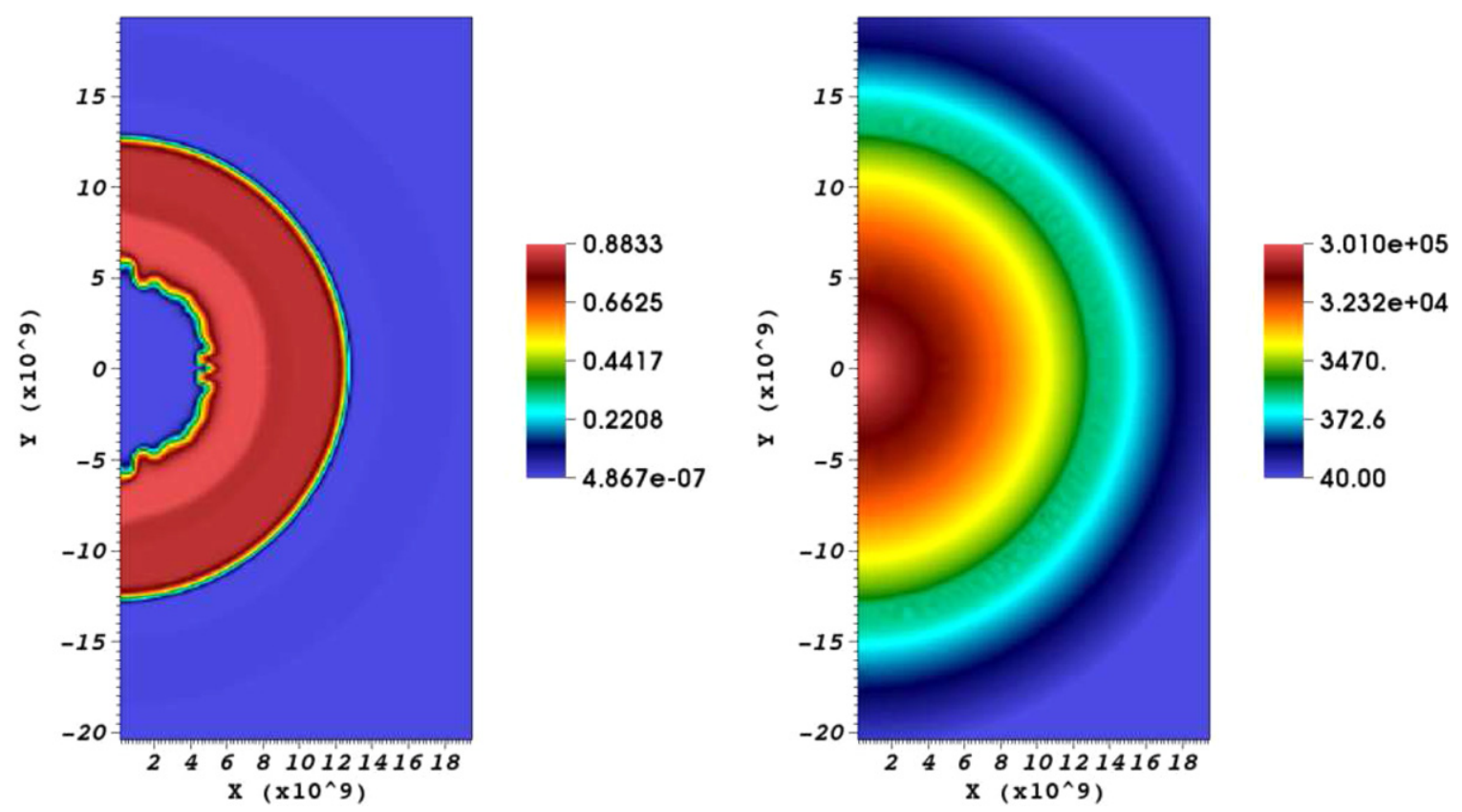

Figure 9. Same as Figure 8 but for PISN model 140sm_rotST $\left(Z=10^{-3} Z_{\odot}\right)$ at time $t=82 \mathrm{~s}$ after the onset of dynamical collapse.

(A color version of this figure is available in the online journal.)

Chen et al. (2011, 2012) and Joggerst \& Whalen (2011). The RT mixing appears to be stronger at the $\mathrm{CO}$ core- $\mathrm{H} / \mathrm{He}$ envelope interface than in the inner regions composed mostly of newly formed ${ }^{56} \mathrm{Ni}$. The other aspect to note in Figures $8-15$ is the significant explosion asymmetry with increased rotation. This can be seen in the left panels of Figures 10 and 14 where the ${ }^{16} \mathrm{O}$ mass fraction maps for the "rotnoST" models are presented. The angular momentum barrier works on the collapse to keep the equatorial material from compressing as much, thus burning less, and hence afterward expanding less rapidly. As a result, the inner core regions where ${ }^{16} \mathrm{O}$ is exhausted and ${ }^{56} \mathrm{Ni}$ has formed in its place take an oblate shape for these models.

Figures 16 and 17 show the ${ }^{56} \mathrm{Ni}$ mass fraction for all models at $\sim 150 \mathrm{~s}$ after maximum compression. The mild RT mixing between the newly formed ${ }^{56} \mathrm{Ni}$ and the remaining ${ }^{16} \mathrm{O}$-rich material, as well as the increased asymmetry with rotation discussed above, are also distinguishable in these figures. The amplified explosion asymmetry with rotation can also be seen in Figures 18 and 19, where the density distributions are plotted for all models at the time the SN shock breaks out of the stellar 

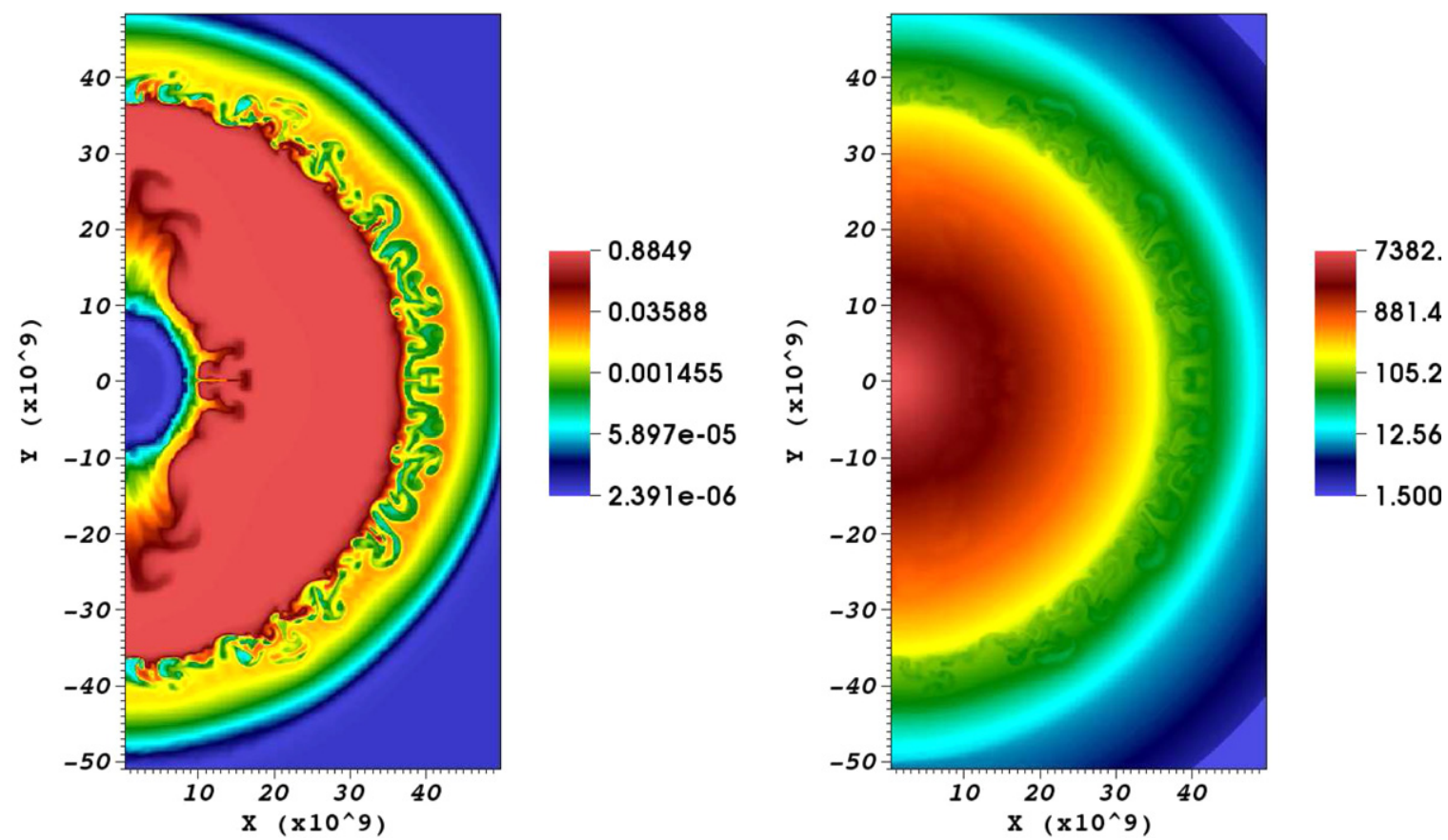

Figure 10. Same as Figure 8 but for PISN model 135sm_rotnoST $\left(Z=10^{-3} Z_{\odot}\right)$ at time $t=138 \mathrm{~s}$ after the onset of dynamical collapse. (A color version of this figure is available in the online journal.)
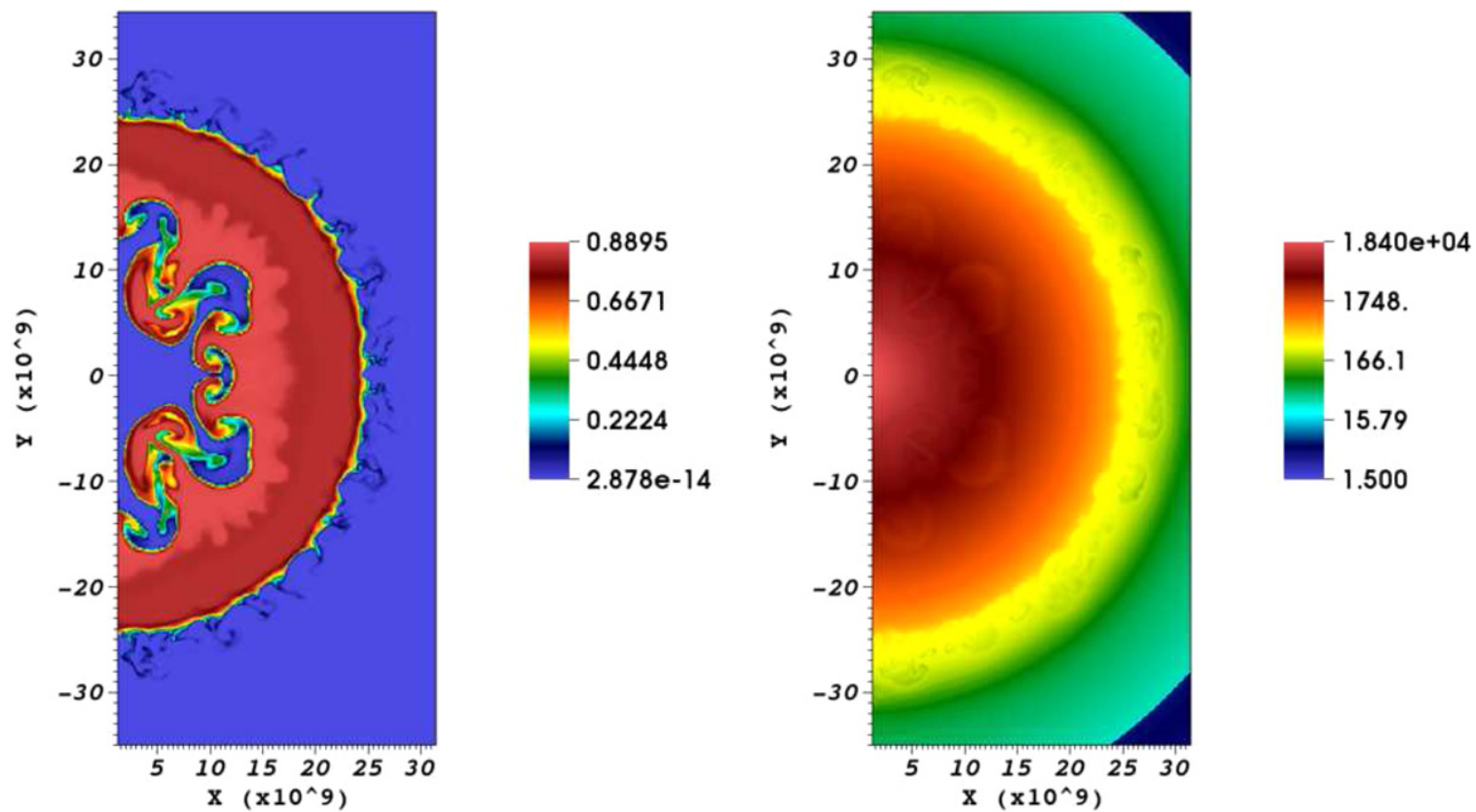

Figure 11. Same as Figure 8 but for PISN model 150sm_rotST_ml2 $\left(Z=10^{-3} Z_{\odot}\right)$ at time $t=112 \mathrm{~s}$ after the onset of dynamical collapse.

(A color version of this figure is available in the online journal.)

surface. The case of the fast rotator $135 \mathrm{sm}$ rotnoST stands out, as the SN blast wave breaks out from the polar regions at an earlier time than from the equator. In addition, the formation of a reverse shock after the SN blast wave breaks out of the stellar envelope leads to the formation of weak RT instability in the outer stellar regions, in accordance with the effects noted by Chen et al. (2011, 2012).

Upon completion of all simulations we were able to calculate the central density and central temperature $\left(\rho_{c}\right.$ and $\left.T_{c}\right)$ hydrodynamic tracks, the total explosion energy, and the total mass of nucleosynthetic products, such as ${ }^{56} \mathrm{Ni}$, for all models. Figure 20 shows the evolution of $\rho_{c}$ and $T_{c}$ upon mapping to
$F L A S H$. At first inspection, the $\rho_{c}-T_{c}$ tracks look very similar among models of the same metallicity series; however, a more detailed analysis reveals that the central values reached in each case are sufficiently different to induce differences in the burning rates, which are all very sensitive to $T$. This is reflected by the final ${ }^{56} \mathrm{Ni}$ mass produced in each case plotted in Figure 21. The PISN explosion energies for all models varied in the range $10^{52}-10^{53}$ erg. Table 2 lists some characteristics of the PISN explosions discussed here.

As can be seen in Figure 21, otherwise structurally identical models produce stronger PISNe with more ${ }^{56} \mathrm{Ni}$ when the rotational velocities are set to zero: "rotnoST_v0" models 

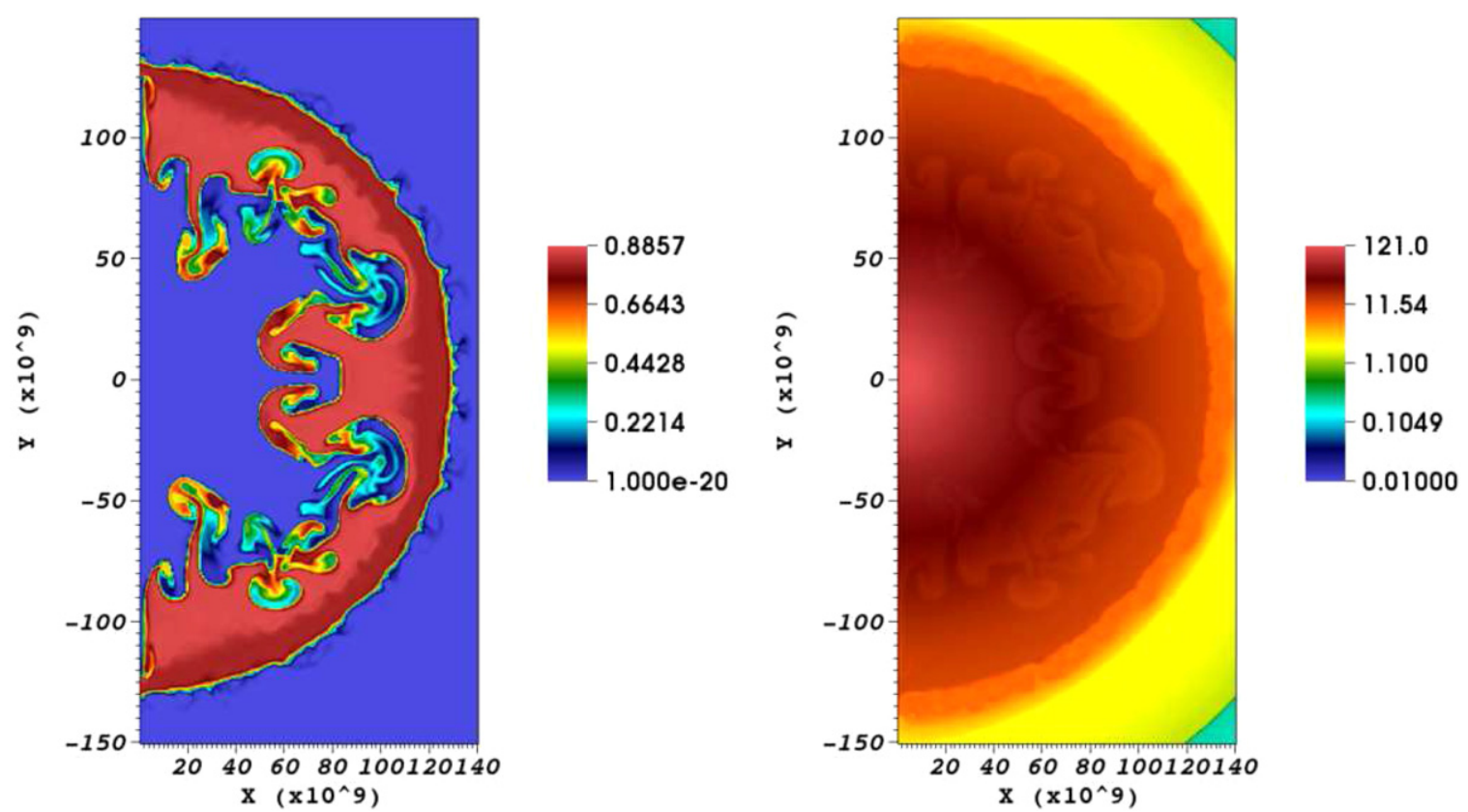

Figure 12. Same as Figure 8 but for PISN model 245sm_norot $\left(Z=10^{-4} Z_{\odot}\right)$ at time $t=245 \mathrm{~s}$ after the onset of dynamical collapse.

(A color version of this figure is available in the online journal.)
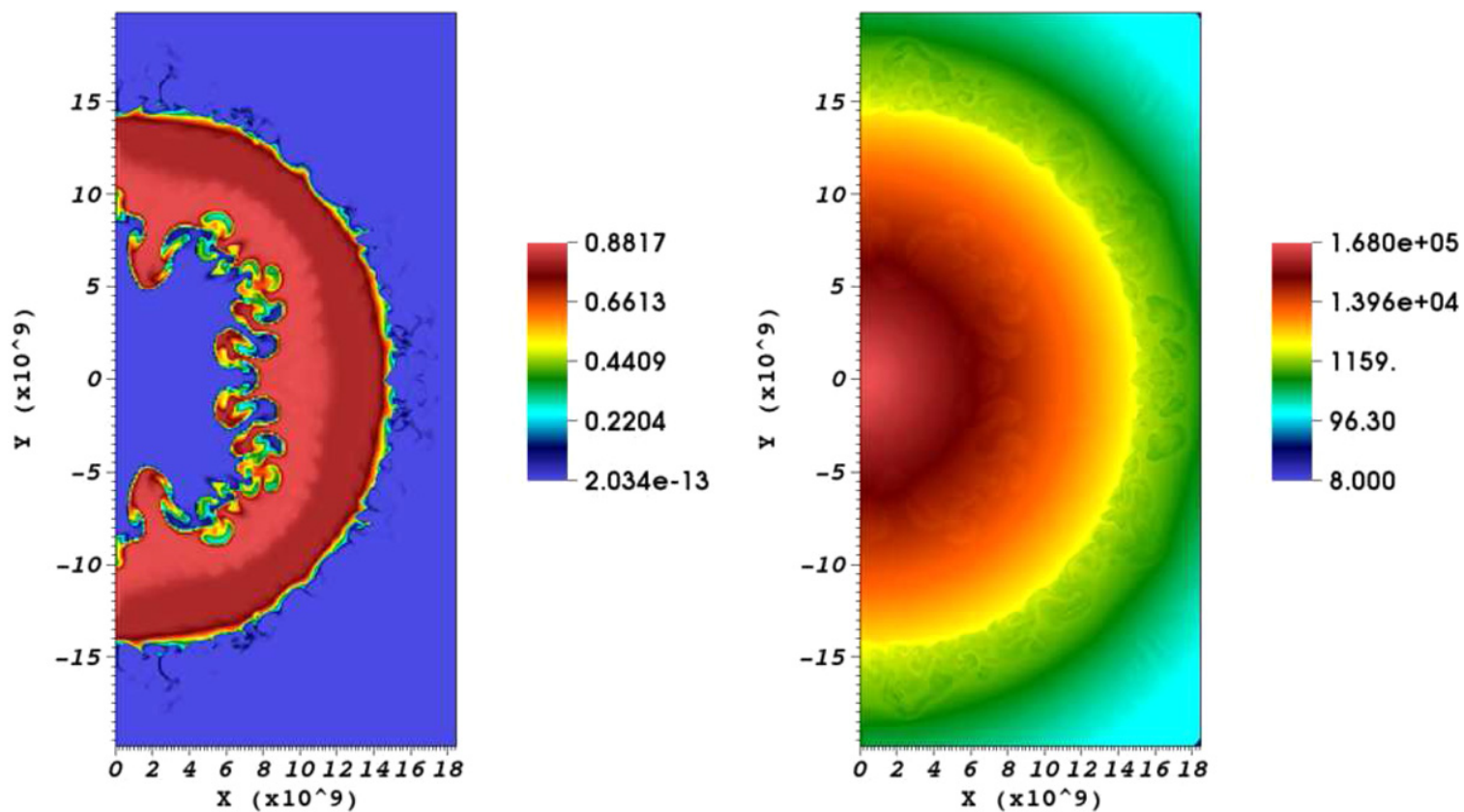

Figure 13. Same as Figure 8 but for PISN model 205sm_rotST $\left(Z=10^{-4} Z_{\odot}\right)$ at time $t=81 \mathrm{~s}$ after the onset of dynamical collapse.

(A color version of this figure is available in the online journal.)

produce $2-10$ times more ${ }^{56} \mathrm{Ni}$ than "rotnoST" models. This is simply because the rotational support from the centrifugal force was artificially removed for the "rotnoST_v0" models prior to mapping to FLASH, thus leading to a stronger collapse. On the other hand, we derive a non-monotonic ${ }^{56} \mathrm{Ni}$ production as a function of CO core rotational velocity for the "norot," "rotST," and "rotnoST" models derived from different evolutionary patterns: adding a small rotation ("rotST" models) seems to lead to more energetic PISNe that produce larger amounts of ${ }^{56} \mathrm{Ni}$, but adding even more rotation leads to much weaker explosions. We discuss this counterintuitive result in Section 3.1.

\subsection{The Source of the Non-monotonic ${ }^{56} \mathrm{Ni}$ Production}

The non-monotonicity of ${ }^{56} \mathrm{Ni}$ production for models of the same $M_{\mathrm{CO}}$ but different rotational profiles holds for both metallicity model series studied here and is a puzzling result. Intuitively, adding rotation to a collapsing star should lead to weaker PISNe because of the presence of an angular momentum barrier that decelerates dynamical collapse, especially in the equatorial regions. Therefore, increasing rotation is expected to lead to smaller $\rho_{c}$ and $T_{c}$ values at maximum compression, lower peak reaction rates, and eventually less ${ }^{56} \mathrm{Ni}$ produced. 

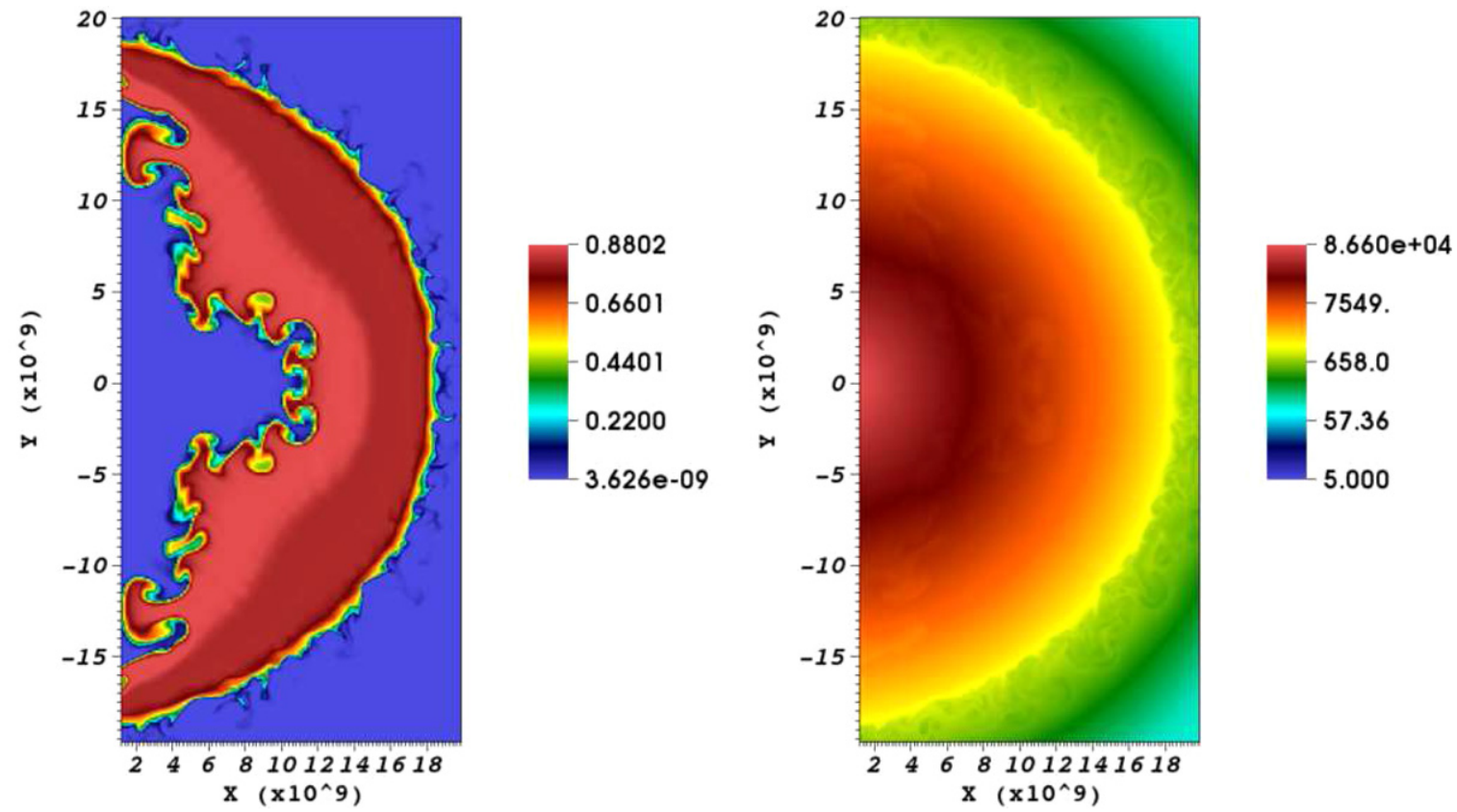

Figure 14. Same as Figure 8 but for PISN model 195sm_rotnoST $\left(Z=10^{-4} Z_{\odot}\right)$ at time $t=80 \mathrm{~s}$ after the onset of dynamical collapse.

(A color version of this figure is available in the online journal.)
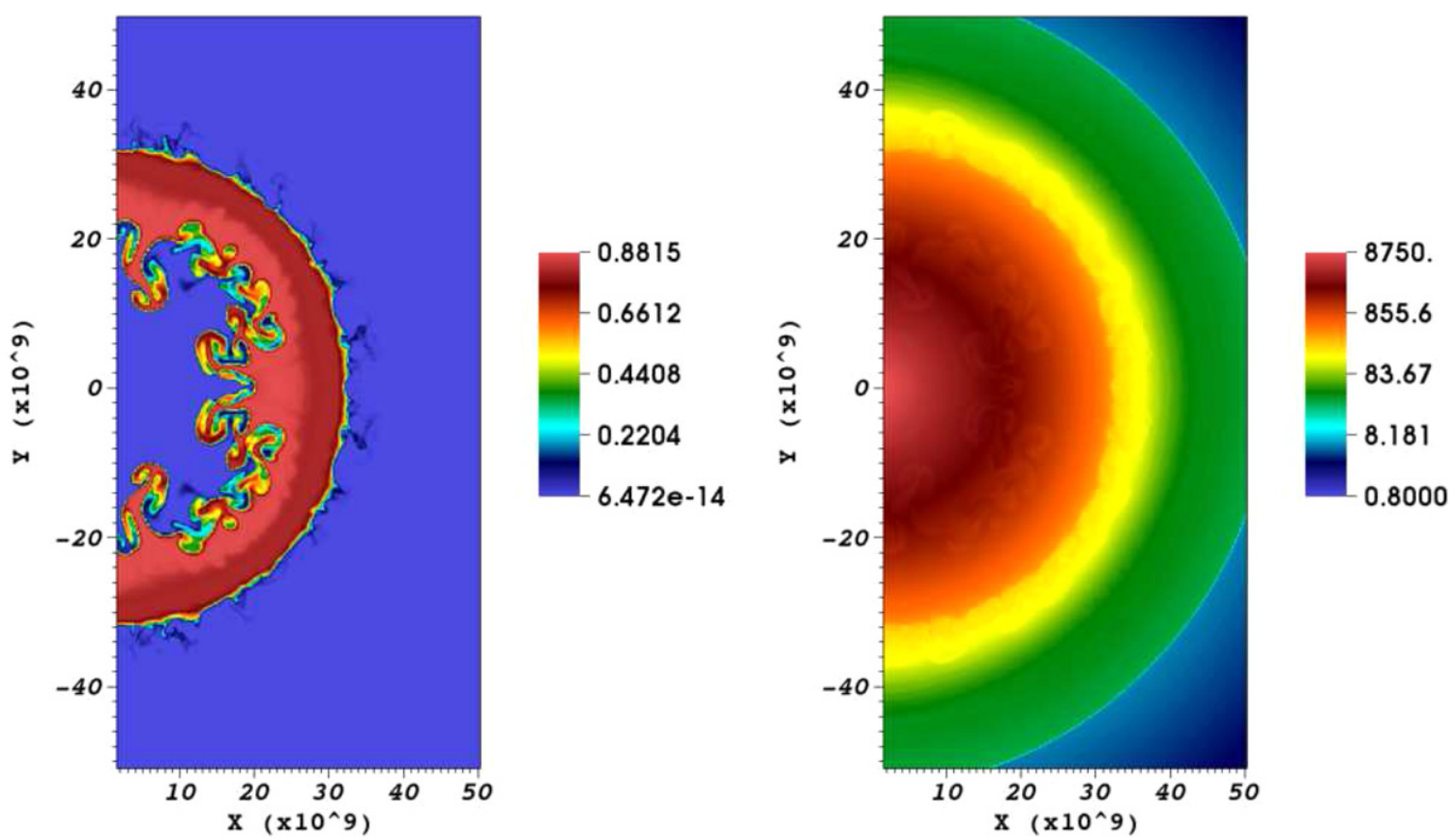

Figure 15. Same as Figure 8 but for PISN model 217sm_rotST_ml2 $\left(Z=10^{-4} Z_{\odot}\right)$ at time $t=105 \mathrm{~s}$ after the onset of dynamical collapse.

(A color version of this figure is available in the online journal.)

To understand the source of the ${ }^{56} \mathrm{Ni}$ non-monotonicity we focus on the "norot" and "rotST" models of both metallicity series. First we discuss the omission of the magnetic field in the mapping of "rotST" models in FLASH. Neglecting the B field corresponds to assuming zero magnetic pressure in the momentum equation that can in turn lead to overestimating the inward acceleration. The MESA evolution allows for the calculation of the radial $\left(B_{r}\right)$ and toroidal $\left(B_{\phi}\right)$ components of the magnetic field in the star using the Spruit (1999, 2002) prescriptions. In the cores of the "rotST" models the total B-field values $\left(B=\left(B_{r}^{2}+B_{\phi}^{2}\right)^{0.5}\right)$ are of the order of $10^{8} \mathrm{G}$, corresponding to magnetic pressures $\left(\sim B^{2} / 4 \pi\right)$ of $\sim 10^{15} \mathrm{dyn}^{-2}$. The total gas and radiation pressure in the same regions are of the order of $\sim 10^{18}-10^{23} \mathrm{dyn} \mathrm{cm}^{-2}$, several orders of magnitude higher, with corresponding plasma parameters $\beta \sim 10^{-3} 10^{-8}$, indicating that the effects of magnetic pressure in the dynamics are negligible.

We then investigate whether multi-dimensional effects, such as large-scale "plume" mixing, the development of hydrodynamical instabilities, and directional effects due to rotation, can be the source of the non-monotonicity. A caveat in this argument is that the dynamical timescales upon mapping to FLASH 

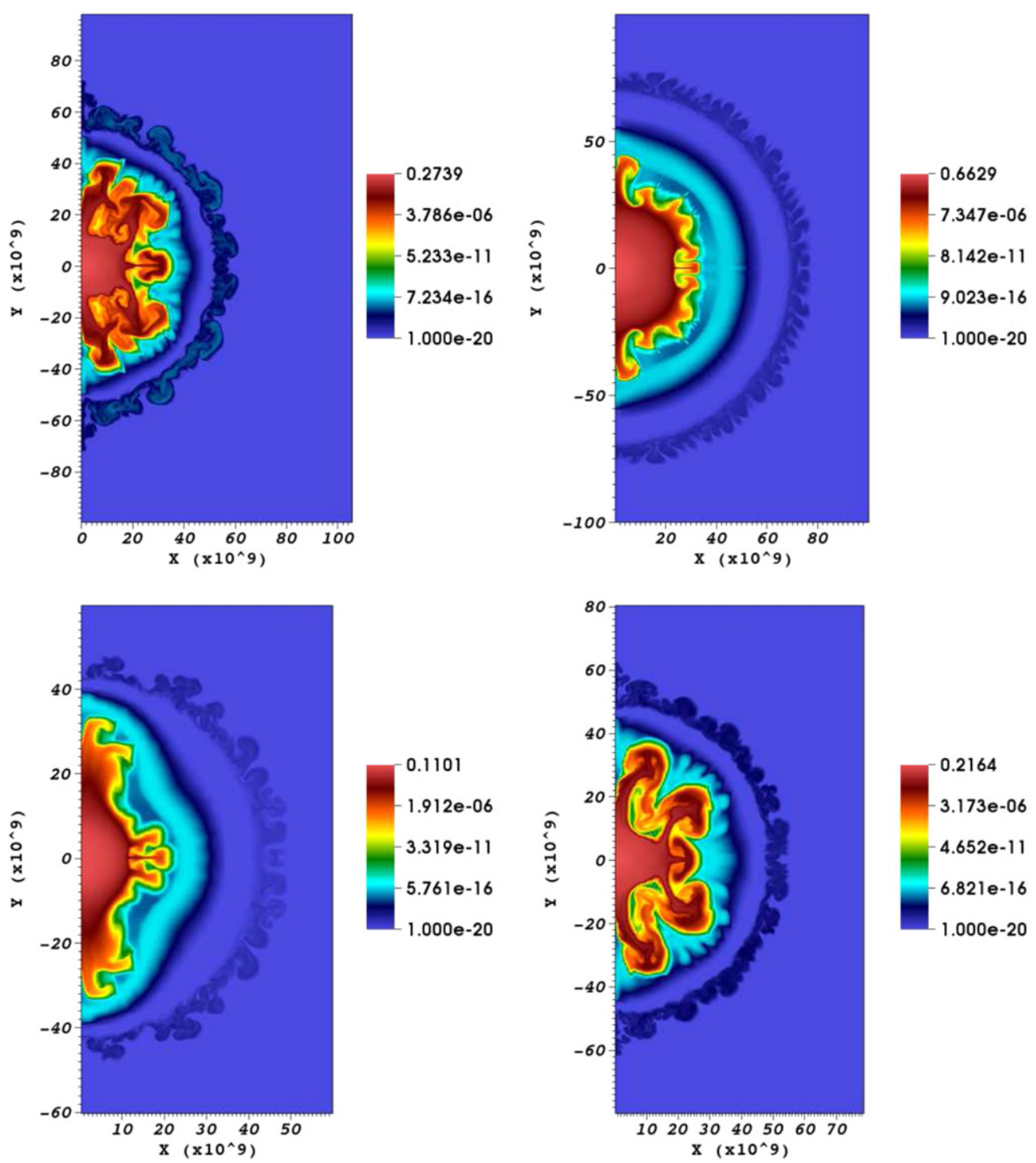

Figure 16. ${ }^{56} \mathrm{Ni}$ mass fraction for the $Z=10^{-3} Z_{\odot}$ PISN model series at $\sim 150 \mathrm{~s}$ after the onset of dynamical collapse: 200sm_norot (upper left), 140sm_rotST (upper right), $135 \mathrm{sm} \_$rotnoST (lower left), and 150sm_rotST_ml2 (lower right).

(A color version of this figure is available in the online journal.)

Table 2

Characteristics of PISN Explosions

\begin{tabular}{|c|c|c|c|c|c|c|c|c|c|c|}
\hline Model & ${ }^{4} \mathrm{He}$ & ${ }^{12} \mathrm{C}$ & ${ }^{16} \mathrm{O}$ & ${ }^{20} \mathrm{Ne}$ & ${ }^{24} \mathrm{Mg}$ & ${ }^{28} \mathrm{Si}$ & ${ }^{32} \mathrm{~S}$ & $\begin{array}{c}\rho_{c, \max , 6} \\
\left(10^{6} \mathrm{~g} \mathrm{~cm}^{-3}\right)\end{array}$ & $\begin{array}{l}T_{c, \max , 9} \\
\left(10^{9} \mathrm{~K}\right)\end{array}$ & ${ }^{56} \mathrm{Ni}_{f}$ \\
\hline \multicolumn{11}{|c|}{$Z=10^{-3} Z_{\odot}$} \\
\hline 200sm_norot & 35.5 & 3.7 & 65.5 & 8.0 & 2.3 & 0.1 & $<0.0001$ & 2.353 & 4.024 & 0.6 \\
\hline 140sm_rotST & 3.8 & 5.0 & 66.3 & 6.7 & 1.6 & 0.1 & $<0.0001$ & 2.879 & 4.126 & 1.4 \\
\hline $135 \mathrm{sm} \_$rotnoST & 7.5 & 4.4 & 66.4 & 7.2 & 1.8 & 0.1 & $<0.0001$ & 2.183 & 3.837 & 0.2 \\
\hline 135sm_rotnoST_v0 & 7.5 & 4.4 & 66.4 & 7.2 & 1.8 & 0.1 & $<0.0001$ & 3.229 & 4.377 & 2.6 \\
\hline 150sm_rotST_ml2 & 14.1 & 4.4 & 65.4 & 6.9 & 1.7 & 0.1 & $<0.0001$ & 2.309 & 3.971 & 0.4 \\
\hline \multicolumn{11}{|c|}{$Z=10^{-4} Z_{\odot}$} \\
\hline 245sm_norot & 37.2 & 4.4 & 80.8 & 10.2 & 3.1 & 0.1 & $<0.0001$ & 3.546 & 4.727 & 9.0 \\
\hline 205sm_rotST & 21.7 & 4.5 & 82.1 & 10.3 & 3.1 & 0.1 & $<0.0001$ & 3.670 & 4.777 & 9.8 \\
\hline 195sm_rotnoST & 20.5 & 4.3 & 81.8 & 10.5 & 3.2 & 0.1 & $<0.0001$ & 3.132 & 4.525 & 5.6 \\
\hline $195 \mathrm{sm}$ rotnoST_v0 & 20.5 & 4.3 & 81.8 & 10.5 & 3.2 & 0.1 & $<0.0001$ & 4.378 & 4.968 & 13.0 \\
\hline 217sm_rotST_ml2 & 22.8 & 4.2 & 80.6 & 10.3 & 3.2 & 0.1 & $<0.0001$ & 3.529 & 4.714 & 8.5 \\
\hline
\end{tabular}

Note. All species masses are in units of $M_{\odot}$ and correspond to the total mass included in the simulation box. 

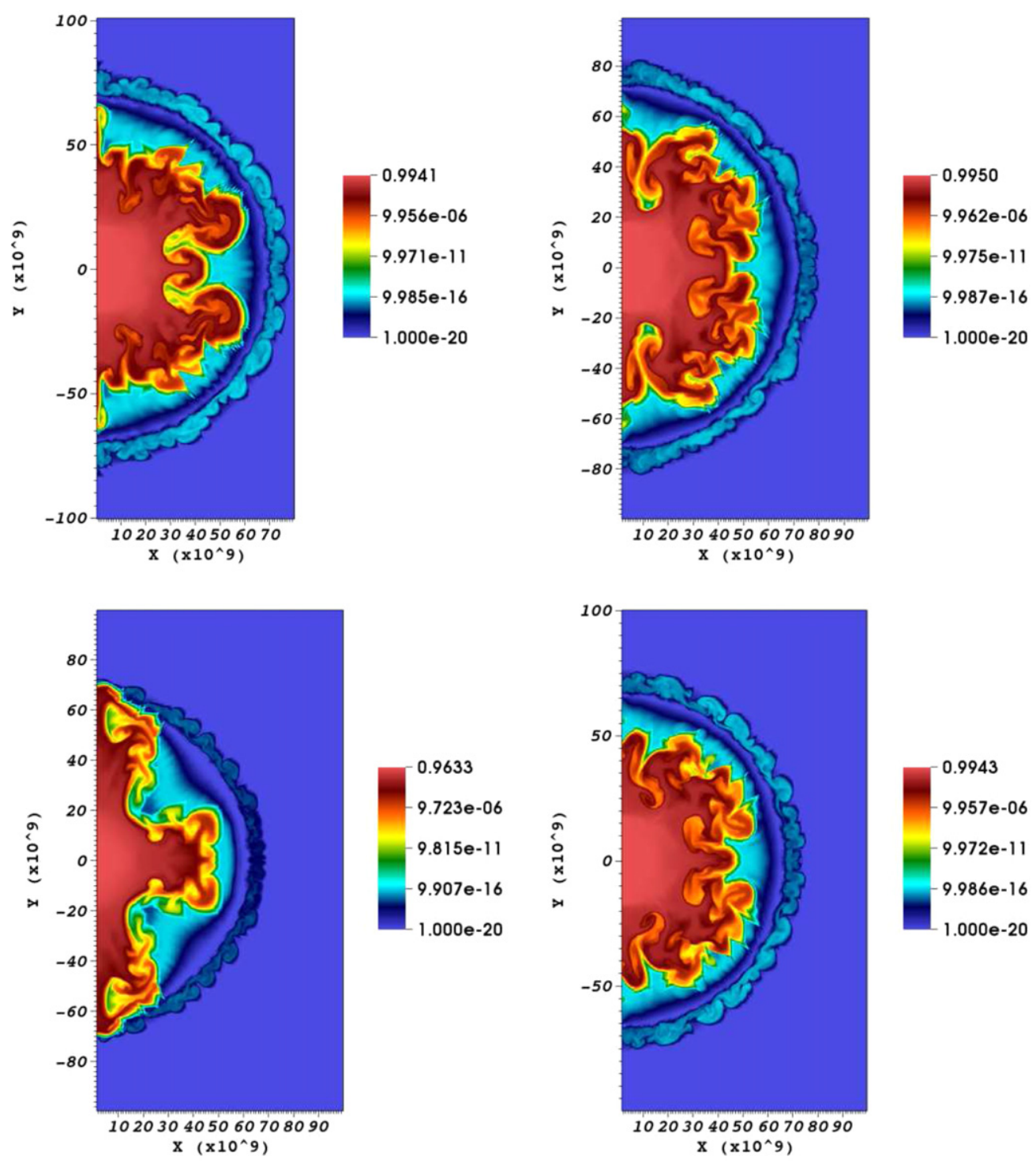

Figure 17. Same as Figure 16 but for the $Z=10^{-4} Z_{\odot}$ PISN model series: $245 \mathrm{sm} \_$norot (upper left), 205sm_rotST (upper right), 195sm_rotnoST (lower left), and 217 sm_rotST_ml2 (lower right).

(A color version of this figure is available in the online journal.)

are likely shorter than relevant 2D mixing timescales, which might have an effect on the dynamics. Nevertheless, we test this hypothesis by re-running the "norot" and "rotST" models in the 1D spherical grid of FLASH with the rotational velocity vector perpendicular to the radial coordinate ("1.5D" treatment). Note that in this case the mapping of the MESA $v_{\text {rot }}$ values to the FLASH grid is straightforward, unlike in the 2D cylindrical mapping scheme we described earlier. The $1.5 \mathrm{D}$ simulations yielded final ${ }^{56} \mathrm{Ni}$ masses very close to those found in the $2.5 \mathrm{D}$ treatment. Therefore the non-monotonic ${ }^{56} \mathrm{Ni}$ production trend with rotational velocity remained unaltered.

Having eliminated multi-dimensional effects and the neglect of B fields as the sources of the dynamical collapse differences between the "norot" and "rotST" models, we turn our attention to structural differences in the initial MESA models. A careful inspection of Figures 1 and 2 indicates that the biggest differences between the initial models are in terms of the radial velocity (see also Section 2). For both metallicity series the "rotST" models seem to collapse with faster speeds. To eliminate this difference, we re-ran the $1.5 \mathrm{D}$ simulations for the "norot" and
"rotST" models with zero initial radial velocity and let FLASH calculate the collapse velocities resulting from the dynamical instability in the core. These simulations yielded somewhat smaller ${ }^{56} \mathrm{Ni}$ masses for all models $\left(0.5 M_{\odot}\right.$ for $200 \mathrm{sm} \_$norot, $1.2 M_{\odot}$ for $145 \mathrm{sm} \_$rotST, $8.8 M_{\odot}$ for $245 \mathrm{sm} \_$norot, and $9.7 M_{\odot}$ for $205 \mathrm{sm} \_$rotST) but the non-monotonicity still remained.

This investigation left us with one remaining option, that the source of non-monotonicity is small initial structural differences in the MESA input models that grow in time with the dynamical collapse. Therefore, we chose to study the first phases of the dynamical collapse of the $1.5 \mathrm{D}$ models in detail and determine in what portions of the cores significant differences develop that lead to different final compressions.

Figures 22 and 23 show the results of this dynamical analysis. The initial acceleration profiles derived using the MESA output are plotted in the top left panels. It can already be seen that the inward acceleration in the outer parts of the $\mathrm{CO}$ core $\left(r \sim 2-5 \times 10^{10} \mathrm{~cm}\right)$ is somewhat higher for the "rotST" models, especially for the $10^{-3} Z_{\odot}$ case. The top right panels show the radial velocity structure for the initial phases of the 

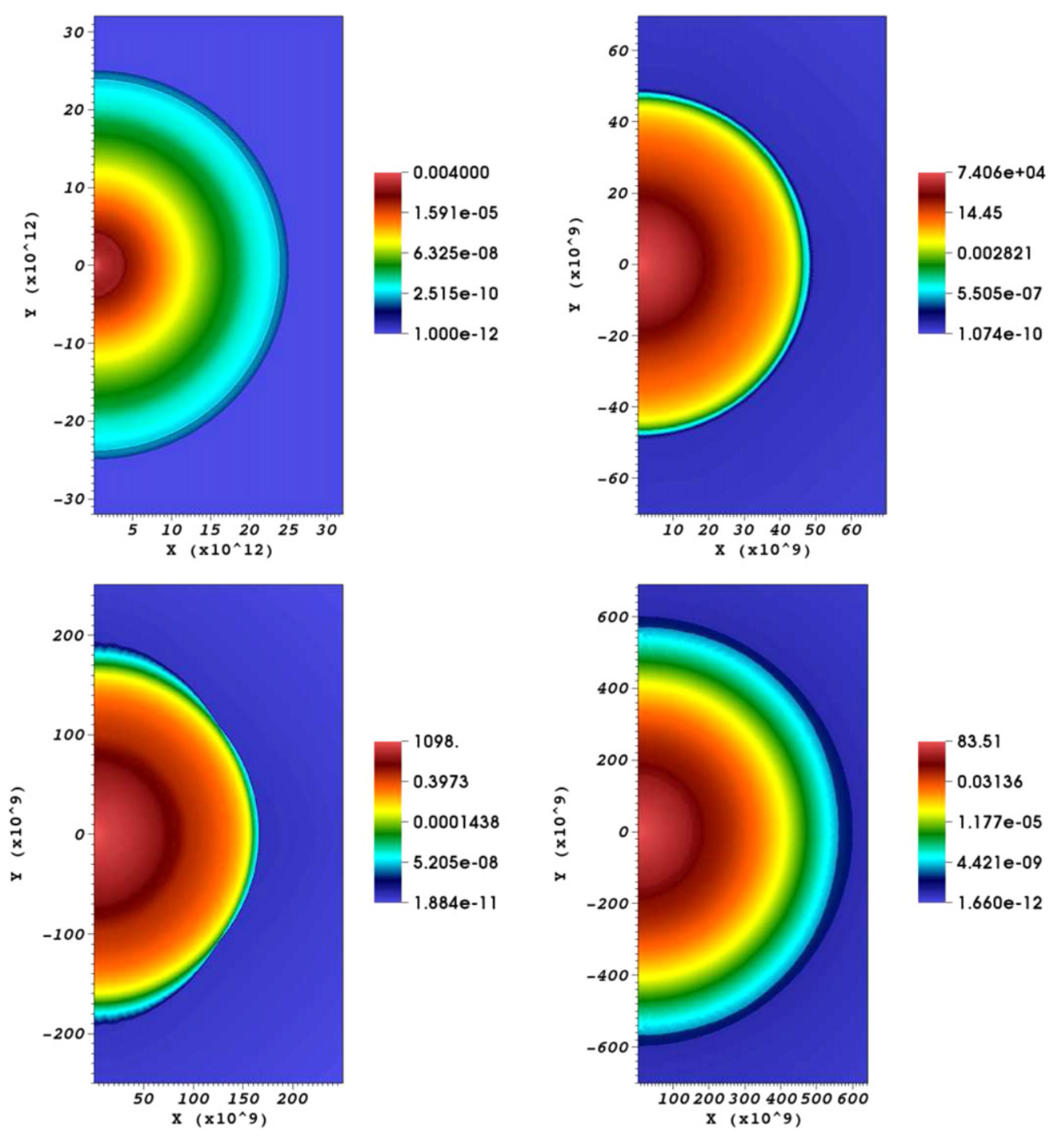

Figure 18. Densities of the $Z=10^{-3} Z_{\odot}$ PISN model series at the time that the SN shock waves break out of the progenitor envelopes.

(A color version of this figure is available in the online journal.)

dynamical collapse $(0-100 \mathrm{~s}$ in steps of $10 \mathrm{~s})$. It is evident that already at 30-40 s the regions of the core outward of $\sim 2 \times 10^{10} \mathrm{~cm}$ collapse somewhat faster for the "rotST" model, especially for the $10^{-3} Z_{\odot}$ model series. This in turn leads to more rapid collapse speeds in the inner regions by $\sim 80-90 \mathrm{~s}$ and, eventually, the production of a stronger SN shock wave at $\sim 10^{10} \mathrm{~cm}$. This suggests that something is different in the dynamical and structural properties between the "norot" and the "rotST" models in the outer core regions $\left(1-6 \times 10^{10} \mathrm{~cm}\right)$.

To further investigate the structural differences between the "norot" and "rotST" models in the outer core regions, we plot their pressure $(P)$ and mean molecular weight $(\mu)$ profiles in the lower panels of Figures 22 and 23. It can be clearly seen that the "rotST" models exhibit steeper pressure gradients at $r>1.5 \times 10^{10} \mathrm{~cm}$ for the $10^{-3} Z_{\odot}$ and $r>5 \times 10^{10} \mathrm{~cm}$ for the $10^{-4} Z_{\odot}$ model series. On the contrary, the $\mu$ gradients in the same outer core regions are flatter for the "rotST" models, an effect that is more clearly visible in the $10^{-3} Z_{\odot}$ case and less so in the $10^{-4} Z_{\odot}$ case. We argue that, for the "rotST" models, the steeper $P$ gradients directly result from the flatter $\mu$ gradients. To support this argument we consider only the contributions of gas $\left(P_{g}=(N / \mu) k T\right.$, where $N$ is the number density and $k$ is the Boltzmann constant) and radiation $\left(P_{r}=a_{r} T^{4}\right.$, where $a_{r}$ is the radiation density constant) to the total pressure and neglect the effects of $e^{+} e^{-}$pairs that are less important in the outer core regions where $\Gamma_{1}>4 / 3$. We then take the gradient of the total pressure:

$$
\frac{d P}{d r}=\frac{1}{\mu} k T \frac{d N}{d r}-N k T \frac{1}{\mu^{2}} \frac{d \mu}{d r}+\frac{N}{\mu} k \frac{d T}{d r}+4 a_{r} T^{3} \frac{d T}{d r} .
$$

This shows that, all else being equal, the steeper the $\mu$ gradient the flatter the $P$ gradient; exactly the effect we see in Figures 22 and 23. Consequently, steeper negative $P$ gradients correspond to higher inward accelerations and radial velocities and more compression in the center.

This result indicates that the source of the non-monotonicity of ${ }^{56} \mathrm{Ni}$ production is the pre-PISN rotational mixing that occurs in the "rotST" models leading to smoother $\mu$ gradients at the $\mathrm{CO}$ core- $\mathrm{H} / \mathrm{He}$ envelope boundaries. In the "norot" models, the boundaries are more well defined in terms of $\mu$, and mixing is minimal, yielding a classic onion structure. The steep $\mu$ gradients at core-envelope interfaces lead to flatter $P$ gradients there and less inward acceleration resulting from the dynamical 

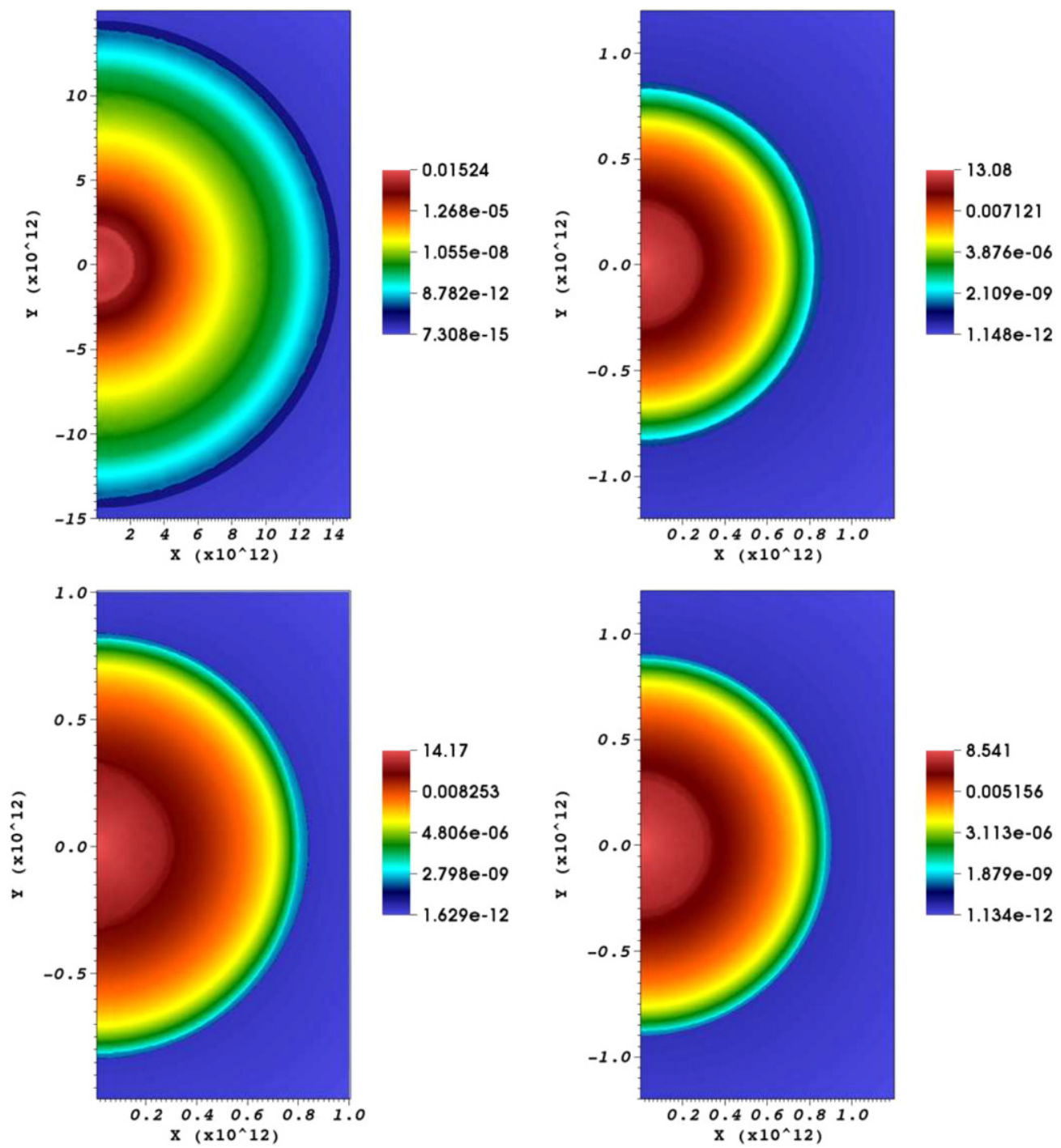

Figure 19. Same as Figure 18 but for the $Z=10^{-4} Z_{\odot}$ PISN model series.

(A color version of this figure is available in the online journal.)
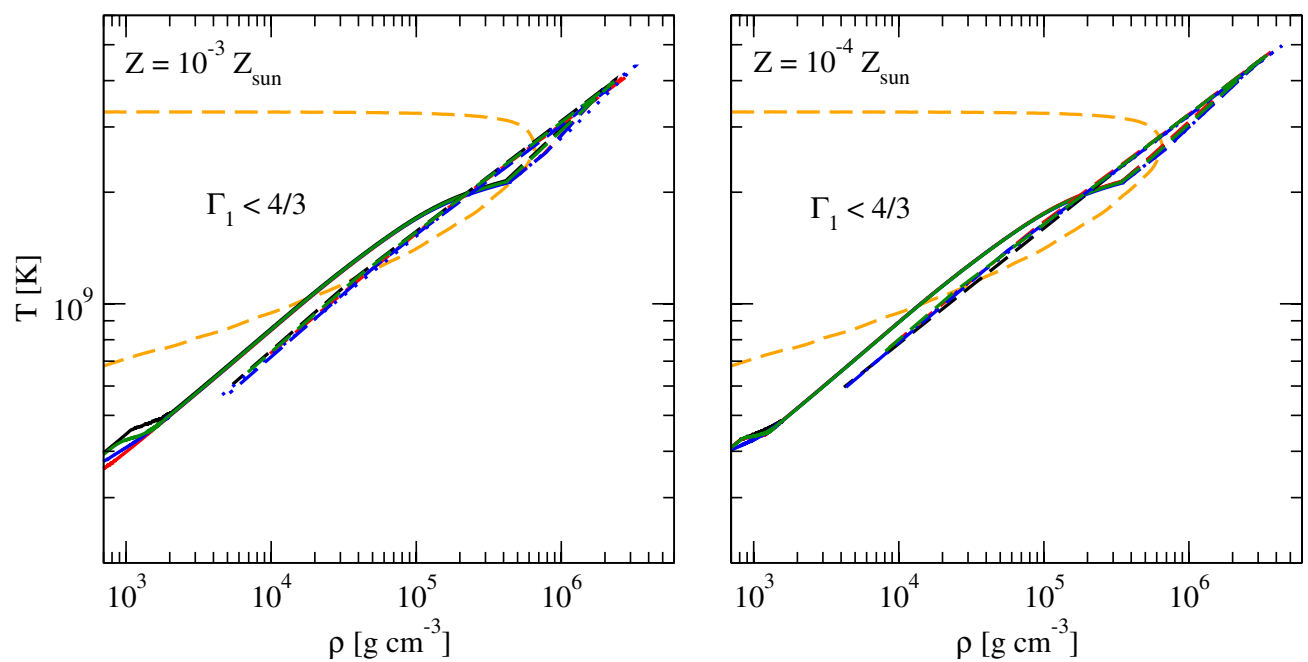

Figure 20. Density-temperature structures of the $Z=10^{-3} Z_{\odot}$ (left) and $Z=10^{-4} Z_{\odot}$ (right) PISN model series. The solid curves represent the $\rho-T$ structure of the models at the time they encounter the dynamical pair instability and are mapped into the 2D FLASH AMR grid. The dashed curves show the subsequent dynamical evolution of $\rho_{c}$ and $T_{c}$. The thick orange dashed curve shows the area of $\Gamma_{1}<4 / 3$ due to $e^{+} e^{-}$pairs. As with Figures $1-6$, black curves represent "norot," red curves the "rotST," blue curves the "rotnoST," and green curves the "rotST_ml2" models. The dotted blue curves represent the rotating models without the effects of ST included, but with their rotational velocities artificially set to zero upon mapping to FLASH ("rotnoST_v0" models).

(A color version of this figure is available in the online journal.) 


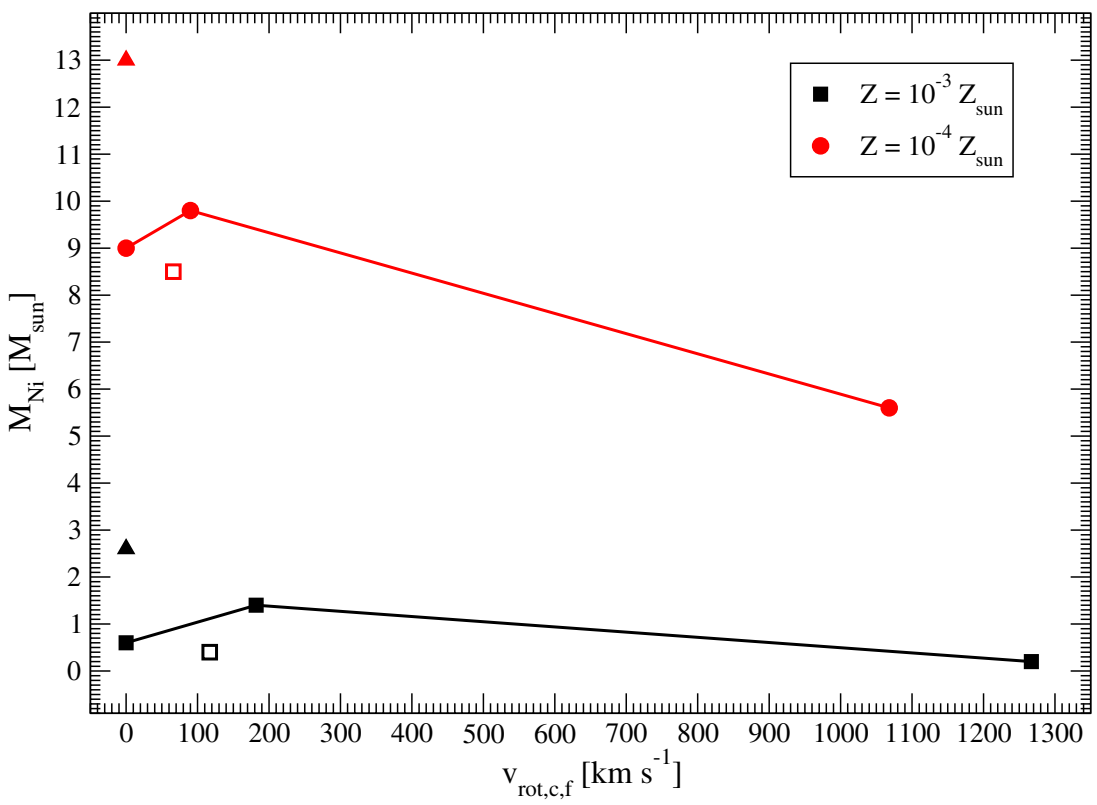

Figure 21. Variation of the final PISN ${ }^{56} \mathrm{Ni}$ mass with $\mathrm{CO}$ core rotational velocity at the time of mapping to FLASH as determined by our set of simulations. The filled triangles show the results for the "rotnoST_v0" models. The open squares show the results for the "rotST_ml2" models.

(A color version of this figure is available in the online journal.)
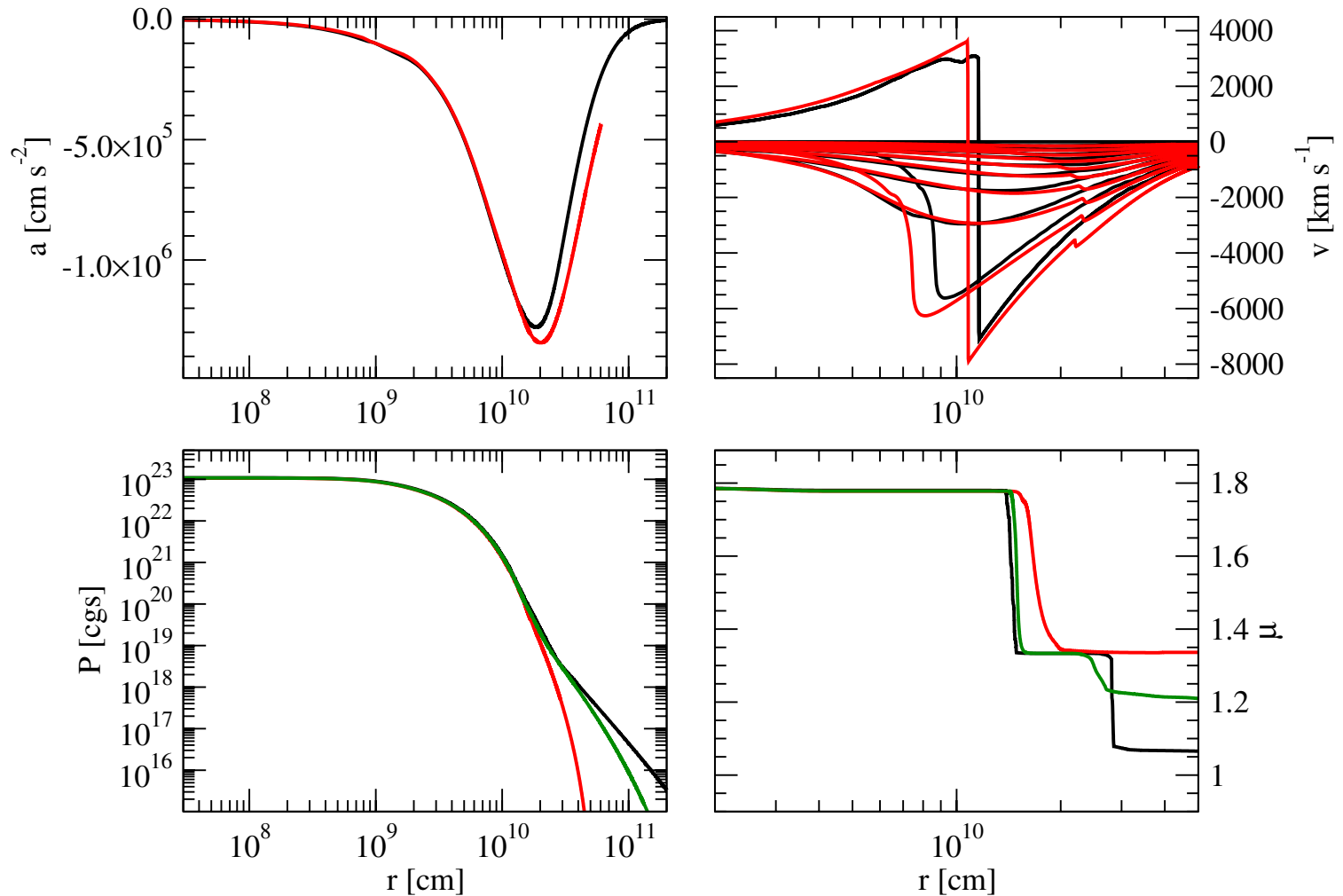

Figure 22. Comparison of the dynamical collapse of the 200sm_norot and the 140sm_rotST models. Acceleration (upper left), radial velocity (upper right), pressure (lower left), and mean molecular weight $\mu$ (lower right) are plotted as a function of radius. The radial velocity is plotted for $0-100 \mathrm{~s}$ in steps of $10 \mathrm{~s}$. Black curves correspond to the "norot," red curves to the "rotST", and green curves to the "rotST_ml2" model.

(A color version of this figure is available in the online journal.)

instability. That leads to less compression in the core and therefore lower $T_{c}$ values reached, slower nuclear reaction rates, and less ${ }^{56} \mathrm{Ni}$ mass formed, for the same initial $M_{\mathrm{CO}}$.

To further support this result, we ran the "rotST_ml2" models for which we adopted a higher mass-loss rate parameter than the standard choice used in the "rotST" models. A high mass-loss rate can lead to less effective rotational mixing, if the timescale for mixing is longer compared to the mass-loss timescale in the outer regions of the progenitor star. This is illustrated by the $\mu$ profiles derived for the "rotST_ml2" models shown as green solid curves in the lower right panels of Figures 22 and 23. The suppression of rotational mixing leads to steep $\mu$ gradients, 

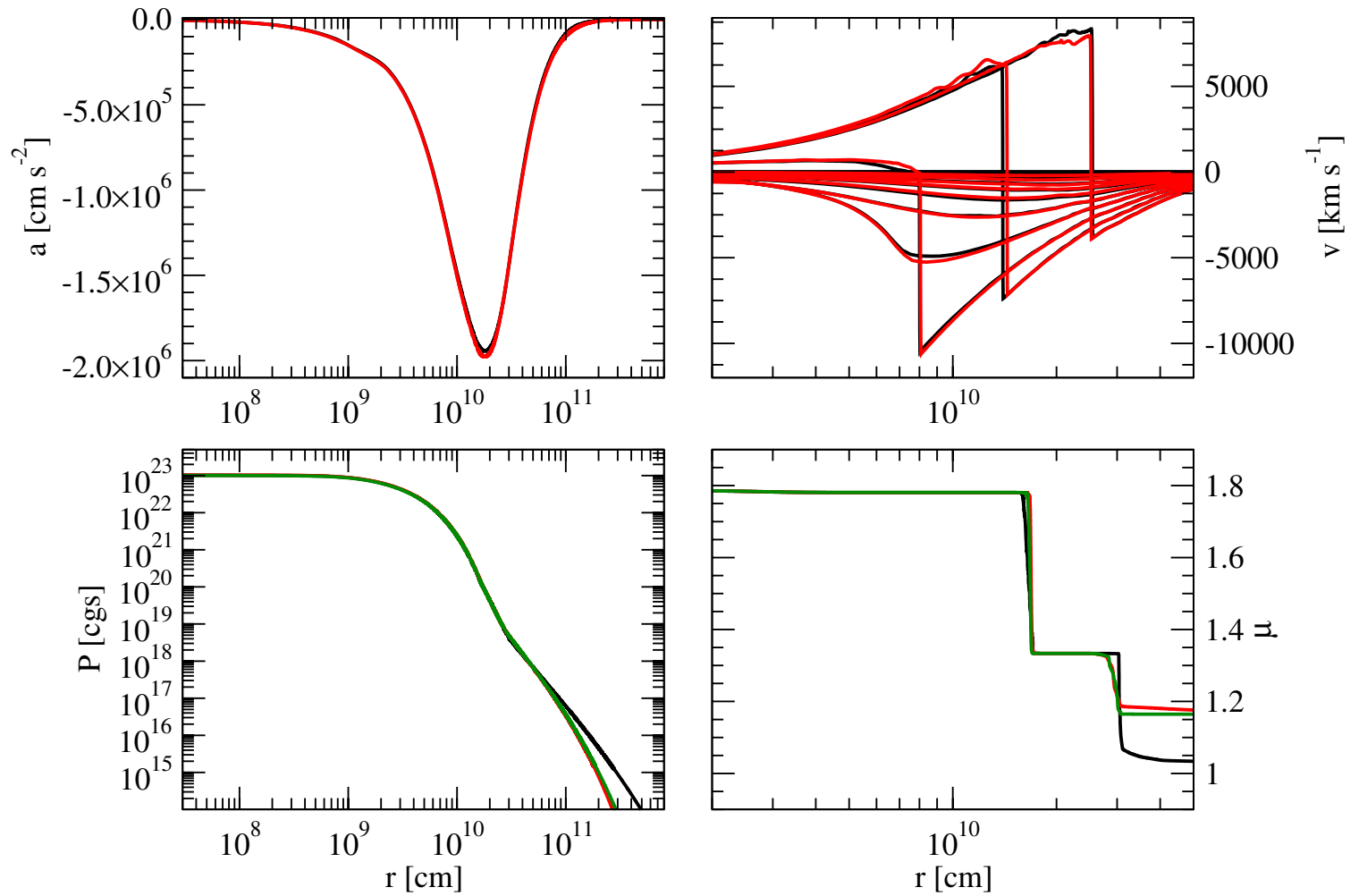

Figure 23. Same as Figure 22 but for the 245sm_norot and the 205sm_rotST models.

(A color version of this figure is available in the online journal.)

similar to those of the "norot" models for CO cores of the same mass. That, in turn, also leads to flatter $P$ gradients than in the case of the "rotST" models and to lower inward acceleration. For the rapidly rotating "rotnoST" models, the angular momentum barrier that develops is strong enough to counter the effects of steeper $P$ gradients and to decelerate dynamical collapse.

The experiment with the "rotST_ml2" models further supports the idea that the source of the ${ }^{56} \mathrm{Ni}$ non-monotonicity is the competition between the effects of rotational mixing in the pre-PISN evolution and the development of an angular momentum barrier for rapid rotation. The effectiveness of the rotational mixing in the pre-PISN MESA evolution is, in turn, dependent on other stellar parameters such as metallicity and mass loss. In the $10^{-4} Z_{\odot}$ model series, mass loss is not as strong as in the $10^{-3} Z_{\odot}$ case and rotational mixing is less effective, but the non-monotonicity still pertains.

\section{SUMMARY AND CONCLUSIONS}

In this work we studied the effects of rotation on PISNe. We used MESA to calculate the evolution of massive (135-245 $\left.M_{\odot}\right)$ low-metallicity $\left(10^{-3}-10^{-4} Z_{\odot}\right)$ stars for two different degrees of ZAMS rotation: $\Omega / \Omega_{c}=0$ and 0.5 . For the rotating models we ran models that include the effects of magnetic fields in the angular momentum transport and the mixing of chemical elements (ST prescriptions), leading to moderately rotating prePISN progenitors ("rotST" models), and models that neglect these effects, leading to rapidly rotating progenitors ("rotnoST" models). We also ran "rotST" models for which a higher massloss rate parameter was adopted ("rotST_ml2" models), which led to the suppression of rotational mixing. The $M E S A$ evolution provided us with PISN progenitor models of the same $M_{\mathrm{CO}}$ for stars in the same metallicity category that were then mapped conservatively in the AMR 2D grid of FLASH including rotation in the direction perpendicular to the grid ("2.5D" treatment). Each PISN explosion was followed with FLASH until after the SN blast wave broke out of the stellar surface. Effects such as the mixing, the energetics, and nucleosynthesis, with emphasis on the production of ${ }^{56} \mathrm{Ni}$ that powers the LCs of PISNe, were studied.

Our 2.5D simulations revealed mild mixing mainly due to the development of RT instability in three different regions: at the burning front between the newly formed ${ }^{56} \mathrm{Ni}$ and the ${ }^{16} \mathrm{O}$ shell, at the interface between the ${ }^{16} \mathrm{O}$ shell and the $\mathrm{He}$ layer, and, lastly, at the reverse shock that develops after the SN shock wave breaks out of the stellar surface into a circumstellar wind material. Rapid rotation also yielded modest explosion asymmetries due to the development of a strong angular momentum barrier: regions close to the equator collapsed with lower speeds than polar ones. This behavior is in agreement with the findings of Glatzel et al. (1985) who also determined larger acceleration along the symmetry axis using their Maclaurin spheroids method. Both the SN blast wave and the distribution of ${ }^{56} \mathrm{Ni}$ were asymmetric for the rapidly rotating "rotnoST" models.

We found a non-monotonic production of ${ }^{56} \mathrm{Ni}$ with increased rotation: non-rotating models produced less ${ }^{56} \mathrm{Ni}$ than slowly rotating "rotST" models, while rapidly rotating "rotnoST" $\mathrm{CO}$ cores produced the smallest amounts among all models, all for the same initial $M_{\mathrm{CO}}$. We determined that the source of the ${ }^{56} \mathrm{Ni}$ production non-monotonicity is the competition between the effects of pre-PISN rotational mixing in the stratification of chemical species in the core and the development of strong centrifugal forces that counter collapse. Effective rotational mixing leads to smooth $\mu$ gradients in the $\mathrm{CO}$ core $-\mathrm{H} / \mathrm{He}$ envelope interface that, in turn, form steep pressure gradients 
corresponding to higher inward acceleration following the $e^{+} e^{-}$pair dynamical instability. This is in contrast with the case of no rotation, where the progenitor star forms a classic onion structure with well-defined boundaries between layers of different composition. Higher inward acceleration leads to more compression in the core, higher central temperatures, increased nuclear reaction rates, and, eventually, larger amounts of ${ }^{56} \mathrm{Ni}$ produced that can power the SN LCs.

The non-monotonic behavior vanishes when the effects of rotational mixing are suppressed (for example, due to increased mass-loss rates preventing effective mixing in the outer parts of the CO cores). For PISN progenitors of the same $M_{\mathrm{CO}}$ and identical structural characteristics, increased rotation leads to less energetic explosions that produce less ${ }^{56} \mathrm{Ni}$. This result is in good agreement with the findings of Glatzel et al. (1985).

Our work is the first in the literature to present 2D simulations of PISNe with rotation. Our results indicate that the final LCs and spectral characteristics of these events are expected to have only modest dependence on initial rotation rates because the differences in the final ${ }^{56} \mathrm{Ni}$ produced are not significant, at least in the case of moderate rotation. In the case of high rotation rates of the $\mathrm{CO}$ core, the PISN explosions are expected to be dim and red due to the small amount of ${ }^{56} \mathrm{Ni}$ produced, and probably to have a long duration LC due to photon diffusion through a large SN ejecta mass. If the first massive stars formed in the universe after the end of the Dark Ages are rapid rotators as predicted in several simulations (Greif et al. 2011; Stacy et al. 2013), then their PISN explosions that missions such as the JWST and WFIRST aim to discover may be somewhat redder than in the case of zero rotation, due to the decreased ${ }^{56} \mathrm{Ni}$ mass. Given that the effectiveness of rotational mixing in the prePISN evolution of low-metallicity stars is unconstrained and dependent upon several stellar parameters, it will be hard to predict the rotational speed of the progenitor star based on the PISN observations alone.

We thank David Dearborn, Christopher Lindner, Daniel Whalen, and Matteo Cantiello for useful discussions and comments. We also thank the Texas Advanced Computing Center for providing us with a generous allocation of computation time on the Stampede supercomputer. This research is supported by the STScI grant AR12820. E.C. thanks the University of Texas Graduate School William C. Powers fellowship for its support of his studies. Some work on this paper was done by J.C.W. in the hospitable clime of the Aspen Center for Physics, which is supported by the NSF Grant PHY-1066293.

\section{REFERENCES}

Abel, T., Anninos, P., Norman, M. L., \& Zhang, Y. 1998, ApJ, 508, 518 Abel, T., Bryan, G. L., \& Norman, M. L. 2000, ApJ, 540, 39 Barkat, Z., Rakavy, G., \& Sack, N. 1967, PhRvL, 18, 379

Bromm, V., Coppi, P. S., \& Larson, R. B. 2002, ApJ, 564, 23 Bromm, V., \& Larson, R. B. 2004, ARA\&A, 42, 79 Brott, I., de Mink, S. E., Cantiello, M., et al. 2011a, A\&A, 530, A115 Brott, I., Evans, C. J., Hunter, I., et al. 2011b, A\&A, 530, A116 Chatzopoulos, E., \& Wheeler, J. C. 2012a, ApJ, 748, 42
Chatzopoulos, E., \& Wheeler, J. C. 2012b, ApJ, 760, 154

Chen, K. J., Heger, A., \& Almgren, A. 2012, in ASP Conf. Ser. 453, Advances in Computational Astrophysics: Methods, Tools, and Outcome, ed. R. CapuzzoDolcetta, M. Limongi, \& A. Tornambè (San Francisco, CA: ASP), 115

Chen, K.-J., Heger, A., \& Almgren, A. S. 2011, CoPhC, 182, 254

Crowther, P. A., Schnurr, O., Hirschi, R., et al. 2010, MNRAS, 408, 731

de Jager, C., Nieuwenhuijzen, H., \& van der Hucht, K. A. 1988, A\&AS, 72,259

de Souza, R. S., Ishida, E. E. O., Johnson, J. L., Whalen, D. J., \& Mesinger, A. 2013, arXiv:1306.4984

Dessart, L., Waldman, R., Livne, E., Hillier, D. J., \& Blondin, S. 2013, MNRAS, 428, 3227

Dubey, A., Reid, L. B., Weide, K., et al. 2009, arXiv:0903.4875

Dufton, P. L., Dunstall, P. R., Evans, C. J., et al. 2011, ApJL, 743, L22

Ekström, S., Georgy, C., Eggenberger, P., et al. 2012, A\&A, 537, A146

Ekström, S., Meynet, G., Chiappini, C., Hirschi, R., \& Maeder, A. 2008, A\&A, 489,685

Fraley, G. S. 1968, Ap\&SS, 2, 96

Fryer, C. L., Woosley, S. E., \& Heger, A. 2001, ApJ, 550, 372

Fryxell, B., Olson, K., Ricker, P., et al. 2000, ApJS, 131, 273

Gal-Yam, A. 2012, Sci, 337, 927

Gal-Yam, A., Mazzali, P., Ofek, E. O., et al. 2009, Natur, 462, 624

Glatzel, W., Fricke, K. J., \& El Eid, M. F. 1985, A\&A, 149, 413

Greif, T. H., Springel, V., White, S. D. M., et al. 2011, ApJ, 737, 75

Heger, A., Langer, N., \& Woosley, S. E. 2000, ApJ, 528, 368

Heger, A., \& Woosley, S. E. 2002, ApJ, 567, 532

Heger, A., Woosley, S. E., \& Spruit, H. C. 2005, ApJ, 626, 350

Hummel, J. A., Pawlik, A. H., Milosavljević, M., \& Bromm, V. 2012, ApJ, 755,72

Joggerst, C. C., \& Whalen, D. J. 2011, ApJ, 728, 129

Kasen, D., Woosley, S. E., \& Heger, A. 2011, ApJ, 734, 102

Kawaler, S. D. 1988, ApJ, 333, 236

Langer, N., Norman, C. A., de Koter, A., et al. 2007, A\&A, 475, L19

Lee, D., Deane, A. E., \& Federrath, C. 2009, in ASP Conf. Ser. 406, Numerical Modeling of Space Plasma Flows: ASTRONUM-2008, ed. N. V. Pogorelov, E. Audit, P. Colella, \& G. P. Zank (San Francisco, CA: ASP), 243

Maeder, A., \& Meynet, G. 2011, arXiv:1109.6171

Meynet, G., Eggenberger, P., \& Maeder, A. 2011, A\&A, 525, L11

Meynet, G., \& Maeder, A. 1997, A\&A, 321, 465

Ober, W. W., El Eid, M. F., \& Fricke, K. J. 1983, A\&A, 119, 61

Pan, T., Kasen, D., \& Loeb, A. 2012, MNRAS, 422, 2701

Paxton, B., Bildsten, L., Dotter, A., et al. 2011, ApJS, 192, 3

Paxton, B., Cantiello, M., Arras, P., et al. 2013, ApJS, 208, 4

Rakavy, G., \& Shaviv, G. 1967, ApJ, 148, 803

Rakavy, G., Shaviv, G., \& Zinamon, Z. 1967, ApJ, 150, 131

Scannapieco, E., Madau, P., Woosley, S., Heger, A., \& Ferrara, A. 2005, ApJ, 633, 1031

Spruit, H. C. 1999, A\&A, 349, 189

Spruit, H. C. 2002, A\&A, 381, 923

Stacy, A., Greif, T. H., \& Bromm, V. 2010, MNRAS, 403, 45

Stacy, A., Greif, T. H., Klessen, R. S., Bromm, V., \& Loeb, A. 2013, MNRAS, 431,1470

Stringfellow, G. S., \& Woosley, S. E. 1988, in Origin and Distribution of the Elements, ed. G. J. Mathews (Singapore: World Scientific), 467

Suijs, M. P. L., Langer, N., Poelarends, A.-J., et al. 2008, A\&A, 481, L87

Timmes, F. X. 1999, ApJS, 124, 241

Timmes, F. X., \& Swesty, F. D. 2000, ApJS, 126, 501

Vink, J. S., de Koter, A., \& Lamers, H. J. G. L. M. 2001, A\&A, 369, 574

Whalen, D. J., Even, W., Frey, L. H., et al. 2012, arXiv:1211.4979

Whalen, D. J., Fryer, C. L., Holz, D. E., et al. 2013, ApJL, 762, L6

Woosley, S. E., Blinnikov, S., \& Heger, A. 2007, Natur, 450, 390

Woosley, S. E., \& Heger, A. 2006, ApJ, 637, 914

Yoon, S.-C., Dierks, A., \& Langer, N. 2012, A\&A, 542, A113

Yoon, S.-C., \& Langer, N. 2005, A\&A, 443, 643

Yoon, S.-C., Woosley, S. E., \& Langer, N. 2010, ApJ, 725, 940

Yusof, N., Hirschi, R., Meynet, G., et al. 2013, MNRAS, 433, 1114

Zahn, J.-P. 1992, A\&A, 265, 115

Zahn, J.-P., Brun, A. S., \& Mathis, S. 2007, A\&A, 474, 145 